\title{
SYMMETRY GROUP ANALYSIS AND INVARIANT SOLUTIONS OF HYDRODYNAMIC-TYPE SYSTEMS
}

\author{
M. B. SHEFTEL
}

Received 25 June 2002

\begin{abstract}
We study point and higher symmetries of systems of the hydrodynamic type with and without an explicit dependence on $t, x$. We consider such systems which satisfy the existence conditions for an infinite-dimensional group of hydrodynamic symmetries which implies linearizing transformations for these systems. Under additional restrictions on the systems, we obtain recursion operators for symmetries and use them to construct infinite discrete sets of exact solutions of the studied equations. We find the interrelation between higher symmetries and recursion operators. Two-component systems are studied in more detail than $n$-component systems. As a special case, we consider Hamiltonian and semi-Hamiltonian systems of Tsarëv.
\end{abstract}

2000 Mathematics Subject Classification: 35Q35, 35L40, 76M60.

1. Introduction. This paper summarizes published and unpublished results of the author on the systems of the hydrodynamic type admitting infinite-dimensional symmetry Lie groups. We study point and higher symmetries, recursion operators, and Hamiltonian structures and use these results for obtaining exact analytical solutions of these equations. Originally Dubrovin and Novikov meant by hydrodynamic-type systems any quasilinear systems of first-order partial differential equations (PDEs) possessing the Hamiltonian structure [4, 5]. Here we consider a more general class of equations of the hydrodynamic type which includes in particular semi-Hamiltonian equations of Tsarëv $[39,40]$ and equations explicitly dependent on $t$ (or $x$ ). Being rich in symmetries, they can be linearized, and infinite sets of exact solutions can be obtained if, in addition, existence conditions for recursion operators are satisfied. Thus, they have as good integrability properties as the Hamiltonian equations.

Systems of the hydrodynamic type describe various physical phenomena such as gas dynamics and hydrodynamics, magnetic hydrodynamics [27], models of nonlinear elasticity and phase transitions [24], chromatography, and electrophoresis equations from physical chemistry and biology [10, 26]. Applications of a different kind are obtained by a representation of the physically interesting higher-order equations as integrability conditions of the hydrodynamic-type systems such as the Euler and Poisson equations of nonlinear acoustics [14] and the Born-Infeld equation of nonlinear electrodynamics [2]. Other applications of hydrodynamic-type systems arise in the theory of averaging nonlinear soliton equations: dispersionless limits of integrable systems [4, 5]. More recent applications use the reductions of nonlinear PDEs to systems of the hydrodynamic type by imposing the dependence of the unknowns on solutions of the hydrodynamictype systems $[8,9,12]$. 
Symmetry group analysis of systems of the hydrodynamic type leads in a natural and algorithmic way to the associated differential geometric structures: metric, connection, curvature, curvilinear orthogonal coordinate systems and their transformations. If, in addition, the Hamiltonian structure exists, then it turns out to be merely an aspect of this geometrical theory $[4,39,40]$. However, the differential geometric theory cannot solve the problem of integration for equations of the hydrodynamic type. We present here another approach to this problem based on a systematic study of higher symmetries and recursion operators [33, 34, 35, 36]. We find the class of such hydrodynamictype systems which admits a infinite set of hydrodynamic symmetries depending on arbitrary solutions of a linear system of PDEs. Formulas for the corresponding invariant solutions determine a linearizing transformation which reduces the problem of obtaining solutions of a nonlinear system of the hydrodynamic type to the problem of solving a linear system. If, in addition, there exist recursion operators mapping symmetries again into symmetries, then they give rise to recursions of hydrodynamic symmetries and, as a consequence, lead to recursions for solutions of the corresponding linear system. Thus, we obtain the recursion formulas which allow us to generate new solutions out of the known "seed" solutions of the original nonlinear system. The action of recursion operators on the hydrodynamic symmetries explicitly dependent on $x, t$ gives rise to infinite discrete sets of higher symmetries which make it possible to find explicitly the corresponding invariant solutions. Thus we obtain infinite discrete sets of exact solutions of the hydrodynamic-type system. They are analogous to the similarity solutions which are well known in the gas and fluid dynamics, and therefore they are expected to describe a physically interesting behavior of the system.

Symmetries, recursions, Hamiltonian structures, and exact solutions of two-component systems of the hydrodynamic type with one space-dimension were studied by the author mainly before Tsarëv's publications [29, 30, 31, 34]. Symmetries, linearizing transformations, and geometric theory of the multicomponent hydrodynamic-type systems explicitly dependent on $x, t$ have been constructed by the author in 1989 [28, 32]. Higher symmetries, higher conservation laws, their interrelations, and the theory of recursion operators for multicomponent hydrodynamic-type systems have been constructed by the author in 1993, 1994, and 1995 [33, 35, 36, 37]. Finally in [13], we further developed the theory of integrability of diagonal hydrodynamic-type systems with an explicit dependence on $t$ or $x$ and presented a nontrivial example of such an integrable system. There we also clarified and formulated precisely the concept of the hydrodynamic symmetries which are first-order symmetries though, generically, they are neither point nor contact symmetries. Therefore, there is no symmetry reduction for solutions invariant with respect to these nonclassical symmetries so that they depend again on two variables.

In Section 2, we study two-component diagonal systems of the hydrodynamic type with an explicit dependence on $t$ or $x$. We find an infinite-dimensional group of their hydrodynamic symmetries and its existence conditions. By considering corresponding invariant solutions, we derive linearizing transformations for these systems. We obtain second-order symmetries and first- and second-order recursion operators with their existence conditions which give rise to infinite discrete sets of exact solutions. 
In Section 3, we study the generalized gas dynamics equations which include, in particular, the equations of the one-dimensional isentropic gas dynamics. We obtain all their hydrodynamic and higher symmetries up to the third order inclusively. We construct first-order recursion operators leading naturally to the Lax representation of these equations, which is used for obtaining explicitly infinite sets of invariant solutions.

In Section 4, we study a particular class of two-component systems which may explicitly depend on $t$ which possesses the Hamiltonian structure, the so-called separable Hamiltonian systems. We obtain their hydrodynamic and higher symmetries, linearizing transformation, second-order recursion operator, Lax representation, and infinite sets of exact solutions.

In Section 5, we consider $n$-component diagonal systems of the hydrodynamic type with no explicit dependence on $t$ and $x$ which admit an infinite-dimensional group of the hydrodynamic symmetries, the so-called semi-Hamiltonian systems. We obtain their hydrodynamic symmetries together with their existence conditions and the associated differential geometric structure. A linearizing transformation, first- and second-order recursion operators, higher symmetries, and infinite sets of exact solutions are constructed.

Finally, in Section 6 , we study $n$-component diagonal hydrodynamic-type systems with an explicit dependence on $t$ or $x$. We obtain their hydrodynamic symmetries and the corresponding linearizing transformations.

2. Symmetries, recursions, and invariant solutions for two-component systems of the hydrodynamic type. Here we study two-component diagonal systems of the hydrodynamic type, which may depend explicitly on the time variable $t$. They are linear homogeneous in the derivatives of unknowns $s(x, t), r(x, t)$ :

$$
s_{t}=\phi(s, r, t) s_{x}, \quad r_{t}=\psi(s, r, t) r_{x},
$$

where $\phi$ and $\psi$ are real-valued functions satisfying nondegeneracy conditions

$$
\phi \neq \psi, \quad \phi_{r}(s, r, t) \psi_{s}(s, r, t) \neq 0,
$$

and the subscripts denote partial derivatives with respect to corresponding variables.

Generalized one-parameter symmetry Lie groups of order $N$ of system (2.1) are generated in an evolutionary (canonical) representation by the Lie equations $[1,15,23]$

$$
\begin{aligned}
& s_{\tau}=f\left(x, t, s, r, s_{x}, r_{x}, \ldots, s_{x}^{(N)}, r_{x}^{(N)}\right), \\
& r_{\tau}=g\left(x, t, s, r, s_{x}, r_{x}, \ldots, s_{x}^{(N)}, r_{x}^{(N)}\right), \quad x_{\tau}=t_{\tau}=0,
\end{aligned}
$$

compatible with (2.1). Here $s=s(x, t, \tau)$ and $r=r(x, t, \tau)$, where $\tau$ is the parameter of a symmetry group whereas $s_{x}^{(N)}=\partial^{N} s / \partial x^{N}$ and $r_{x}^{(N)}=\partial^{N} r / \partial x^{N}$.

Compatibility conditions for (2.1) and (2.3), $s_{\tau t}=s_{t \tau}$ and $r_{\tau t}=r_{t \tau}$, take the form of the determining equations for the symmetry characteristics $(f, g)$ :

$$
\begin{aligned}
& D_{t}[f]-\phi D_{x}[f]-s_{x}\left(\phi_{s} f+\phi_{r} g\right)=0, \\
& D_{t}[g]-\psi D_{x}[g]-r_{x}\left(\psi_{s} f+\psi_{r} g\right)=0,
\end{aligned}
$$


where $D_{t}$ and $D_{x}$ are the operators of total derivatives with respect to $t$ and $x$ :

$$
\begin{aligned}
& D_{x}=\frac{\partial}{\partial x}+s_{x} \frac{\partial}{\partial s}+r_{x} \frac{\partial}{\partial r}+\sum_{k=1}^{\infty}\left(s_{x}^{(k+1)} \frac{\partial}{\partial s_{x}^{(k)}}+r_{x}^{(k+1)} \frac{\partial}{\partial r_{x}^{(k)}}\right), \\
& D_{t}=\frac{\partial}{\partial t}+\phi s_{x} \frac{\partial}{\partial s}+\psi r_{x} \frac{\partial}{\partial r}+\sum_{k=1}^{\infty}\left(D_{x}^{k}\left[\phi s_{x}\right] \frac{\partial}{\partial s_{x}^{(k)}}+D_{x}^{k}\left[\psi r_{x}\right] \frac{\partial}{\partial r_{x}^{(k)}}\right),
\end{aligned}
$$

and $D_{t}$ is calculated with the use of system (2.1).

2.1. Hydrodynamic symmetries of diagonal systems with explicit time dependence. For hydrodynamic symmetries, $N=1$ in (2.3) and (2.4). Solving (2.4), we obtain the following results. Introduce four functions of one variable $b(s), d(r), \Phi_{0}(s)$, and $\Theta_{0}(r)$ and define the functions $\Phi(s, r, t), \Theta(s, r, t), \hat{\Phi}(s, r, t), \hat{\Theta}(s, r, t), \hat{\phi}(s, r, t)$, and $\hat{\psi}(s, r, t)$ by the formulas

$$
\begin{aligned}
\Phi_{r}(s, r, t) & =\frac{\phi_{r}(s, r, t)}{(\phi-\psi)}, \\
\Theta_{s}(s, r, t) & =\frac{\psi_{r}(s, r, t)}{(\psi-\phi)}, \\
\hat{\Phi}(s, r, t) & =b(s) \Phi_{s}(s, r, t)+d(r) \Phi_{r}(s, r, t)+\Phi_{0}(s), \\
\hat{\Theta}(s, r, t) & =b(s) \Theta_{s}(s, r, t)+d(r) \Theta_{r}(s, r, t)+\Theta_{0}(r), \\
\hat{\phi}(s, r, t) & =b(s) \phi_{s}(s, r, t)+d(r) \phi_{r}(s, r, t), \\
\hat{\psi}(s, r, t) & =b(s) \psi_{s}(s, r, t)+d(r) \psi_{r}(s, r, t) .
\end{aligned}
$$

DeFINITION 2.1. We call (2.1) a generic system (with respect to the hydrodynamic symmetries) if its coefficients $\phi, \psi$ do not satisfy the following constraints:

$$
\phi_{t}=\beta(t) \phi^{2}+\varepsilon(t) \phi+\lambda(t), \quad \psi_{t}=\beta(t) \psi^{2}+\varepsilon(t) \psi+\lambda(t),
$$

with arbitrary smooth functions $\beta(t), \varepsilon(t)$, and $\lambda(t)$.

Results of the symmetry analysis are summarized in the following theorem [34].

THEOREM 2.2. A diagonal two-component generic system (2.1) of the hydrodynamic type, which may explicitly depend on $t$ admits an infinite set of hydrodynamic symmetries with a functional arbitrariness if and only if the following two conditions are satisfied:

(1) coefficients $\phi, \psi$ of system (2.1) satisfy the equations

$$
\Phi_{r t}=\beta \phi_{r}, \quad \Theta_{s t}=\beta \psi_{s},
$$

where $\beta$ is an arbitrary real constant, $\Phi(s, r, t)$ and $\Theta(s, r, t)$ are determined by (2.6), and partial derivatives with respect to $t$ are taken at constant values of $s$ and $r$;

(2) there exist four functions of one variable $b(s), d(r), \Phi_{0}(s)$, and $\Theta_{0}(r)$ satisfying the equations

$$
\hat{\Phi}_{r}=\Phi_{r}(\hat{\Phi}-\hat{\Theta}), \quad \hat{\Theta}_{s}=\Theta_{s}(\hat{\Theta}-\hat{\Phi}),
$$

with $\hat{\Phi}(s, r, t)$ and $\hat{\Theta}(s, r, t)$ defined by (2.7). 
The symmetries in this theorem are generated by the Lie equations

$$
\begin{gathered}
s_{\tau}=f=\tilde{\phi}(x, t, s, r) s_{x}+b(s), \quad r_{\tau}=g=\tilde{\psi}(x, t, s, r) r_{x}+d(r), \\
\tilde{\phi}(x, t, s, r)=a(s, r) \exp \left\{\beta\left[x+\int_{0}^{t} \phi(s, r, t) d t\right]\right\}+\frac{1}{\beta} \hat{\Phi}(s, r, t) \\
\tilde{\psi}(x, t, s, r)=c(s, r) \exp \left\{\beta\left[x+\int_{0}^{t} \psi(s, r, t) d t\right]\right\}+\frac{1}{\beta} \hat{\Theta}(s, r, t) \\
\tilde{\phi}(x, t, s, r)=a(s, r)+\int_{0}^{t} \hat{\phi}(s, r, t) d t-\hat{\Phi}(s, r)\left[x+\int_{0}^{t} \phi(s, r, t) d t\right] \\
\tilde{\psi}(x, t, s, r)=c(s, r)+\int_{0}^{t} \hat{\psi}(s, r, t) d t-\hat{\Theta}(s, r)\left[x+\int_{0}^{t} \psi(s, r, t) d t\right]
\end{gathered}
$$

Here the integrals with respect to $t$ are taken at constant values of $s$ and $r$ and the functions $\hat{\phi}, \hat{\psi}$ are defined by formulas (2.8). The functions $a(s, r)$ and $c(s, r)$ form an arbitrary smooth solution of the linear system

$$
a_{r}(s, r)=\Phi_{r}(s, r, 0)(a-c), \quad c_{s}(s, r)=\Theta_{s}(s, r, 0)(c-a) .
$$

REMARK 2.3. We can use the freedom in definition (2.6) of the functions $\Phi, \Theta$ to transform (2.10) to a more simple form

$$
\Phi_{t}(s, r, t)=\beta \phi(s, r, t), \quad \Theta_{t}(s, r, t)=\beta \psi(s, r, t) .
$$

REMARK 2.4. Solution manifold of the linear system (2.15) is locally parameterized by two arbitrary functions of one variable $c_{1}(s)$ and $c_{2}(r)$. They determine a functional arbitrariness in the hydrodynamic symmetries (2.12), (2.13), and (2.14) of system (2.1).

System (2.15) has the trivial solution $a(s, r)=c(s, r)=c_{0}=$ const.

Condition (2) of Theorem 2.2 always has the trivial solution

$$
b(s)=d(r)=0, \quad \Phi_{0}(s)=\Theta_{0}(r)=c_{0}=\text { const }, \quad \hat{\Phi}=\hat{\Theta}=c_{0}, \quad \hat{\phi}=\hat{\psi}=0 .
$$

COROLLARY 2.5. Condition (1) of Theorem 2.2 is necessary and sufficient for system (2.1) to admit an infinite set of hydrodynamic symmetries generated by the Lie equations

$$
s_{\tau}=\tilde{\phi}(x, t, s, r) s_{x}, \quad r_{\tau}=\tilde{\psi}(x, t, s, r) r_{x}
$$

which are linear homogeneous in the derivatives with the coefficients $\tilde{\phi}, \tilde{\psi}$ defined as

$$
\begin{aligned}
& \tilde{\phi}(x, t, s, r)=a(s, r) \exp \left\{\beta\left[x+\int_{0}^{t} \phi(s, r, t) d t\right]\right\}+c_{0} \quad \text { for } \beta \neq 0, \\
& \tilde{\psi}(x, t, s, r)=c(s, r) \exp \left\{\beta\left[x+\int_{0}^{t} \psi(s, r, t) d t\right]\right\}+c_{0} \\
& \tilde{\phi}(x, t, s, r)=a(s, r)+c_{0}\left[x+\int_{0}^{t} \phi(s, r, t) d t\right] \\
& \tilde{\psi}(x, t, s, r)=c(s, r)+c_{0}\left[x+\int_{0}^{t} \psi(s, r, t) d t\right]
\end{aligned}
$$


REMARK 2.6. Condition (1) of Theorem 2.2 with $\beta=0$ is satisfied in particular for system (2.1) with the coefficients $\phi(s, r)$ and $\psi(s, r)$ with no explicit dependence on $t$. Such a system always has an infinite set of hydrodynamic symmetries with a functional arbitrariness. In this case, the coefficients (2.20) of the Lie equations become

$$
\begin{aligned}
& \tilde{\phi}(x, t, s, r)=a(s, r)+c_{0}[x+t \phi(s, r)], \\
& \tilde{\psi}(x, t, s, r)=c(s, r)+c_{0}[x+t \psi(s, r)] .
\end{aligned}
$$

REMARK 2.7. Condition (2) of Theorem 2.2 is an additional constraint which provides the existence of symmetries, with the Lie equations (2.12) linear inhomogeneous in derivatives. Every nontrivial solution of (2.11) generates such symmetries.

2.2. Infinite-dimensional Lie algebra of hydrodynamic symmetries and recursions of symmetries. Let system (2.1) possess two one-parameter symmetry groups generated by the Lie equations (2.12) and by the Lie equations of the same form with another parameter $\bar{\tau}$ :

$$
s_{\bar{\tau}}=\bar{f}=\bar{\phi}(x, t, s, r) s_{x}+\bar{b}(s), \quad r_{\bar{\tau}}=\bar{g}=\bar{\psi}(x, t, s, r) r_{x}+\bar{d}(r) .
$$

Here the coefficients $\bar{\phi}, \bar{\psi}$ are determined by formulas (2.13) or (2.14) for $\beta \neq 0$ or for $\beta=0$, respectively, with the following change of notation:

$$
\begin{aligned}
(a(s, r), c(s, r)) & \longmapsto(\overline{\bar{a}}(s, r), \bar{c}(s, r)), \\
(\hat{\Phi}(s, r, t), \hat{\Theta}(s, r, t)) & \longmapsto(\hat{\bar{\Phi}}(s, r, t), \hat{\bar{\Theta}}(s, r, t)), \\
(\phi(s, r, t), \psi(s, r, t)) & \longmapsto(\hat{\bar{\phi}}(s, r, t), \hat{\bar{\psi}}(s, r, t)),
\end{aligned}
$$

where the functions $\hat{\bar{\Phi}}$, $\hat{\bar{\Theta}}$ are defined by formulas (2.7) and the functions $\hat{\bar{\phi}}, \hat{\bar{\psi}}$ are defined by formulas (2.8) with the change of $b(s), d(r), \Phi_{0}(s)$, and $\Theta_{0}(r)$ to $\bar{b}(s), \bar{d}(r)$, $\bar{\Phi}_{0}(s)$, and $\bar{\Theta}_{0}(r)$, respectively. Here the functions $\hat{\bar{\Phi}}, \hat{\bar{\Theta}}$ must satisfy (2.11) in condition (2) of Theorem 2.2 and the functions $\bar{a}(s, r), \bar{c}(s, r)$ form an arbitrary smooth solution of the linear system (2.15).

Let $\sigma=(f, g)$ and $\bar{\sigma}=(\bar{f}, \bar{g})$ be characteristics of the symmetries (2.12) and (2.22). A higher symmetry generator with the characteristic $\sigma$ is defined as follows [23]:

$$
\begin{aligned}
\hat{X}_{\sigma}= & f \frac{\partial}{\partial s}+g \frac{\partial}{\partial r}+\left(D_{t}[f]\right) \frac{\partial}{\partial s_{t}} \\
& +\left(D_{t}[g]\right) \frac{\partial}{\partial r_{t}}+\sum_{N=1}^{\infty}\left\{\left(D_{x}^{N}[f]\right) \frac{\partial}{\partial s_{x}^{(N)}}+\left(D_{x}^{N}[g]\right) \frac{\partial}{\partial r_{x}^{(N)}}\right\},
\end{aligned}
$$

where the operator $D_{t}$ of the total derivative with respect to $t$ is calculated with the use of (2.1). The formula for $\hat{X}_{\bar{\sigma}}$ is obtained by the substitution of $(\bar{f}, \bar{g})$ for $(f, g)$ in formula (2.24).

The usual Lie commutator $\left[\hat{X}_{\bar{\sigma}}, \hat{X}_{\sigma}\right]=\hat{X}_{[[\sigma, \bar{\sigma}]]} \equiv \hat{X}_{\tilde{\sigma}}$ generates the commutator of symmetry characteristics

$$
\tilde{\sigma}=[[\sigma, \bar{\sigma}]]=\sigma^{\prime}[\bar{\sigma}]-\bar{\sigma}^{\prime}[\sigma]
$$


with $\sigma^{\prime}$ denoting the operator of the Frechét derivative [11]:

$$
\left(\sigma^{\prime}\right)_{\alpha \beta}=\sum_{j=0}^{\infty} \frac{\partial \sigma_{\alpha}}{\partial u_{x}^{\beta(j)}} D_{x}^{j}, \quad \alpha, \beta=1,2,
$$

where $\sigma_{1}=f, \sigma_{2}=g, u^{1}=s$, and $u^{2}=r$. The commutator (2.25) of symmetry characteristics for system (2.1) is again a characteristic $\tilde{\sigma}=(\tilde{f}, \tilde{g})$ of some symmetry for this system generated by the Lie equations with the parameter $\tilde{\tau}: s_{\tilde{\tau}}=\tilde{f}, r_{\tilde{\tau}}=\tilde{g}$, where

$$
\begin{aligned}
& \tilde{f}=[[\sigma, \bar{\sigma}]]_{1}=\bar{f} \frac{\partial f}{\partial s}-f \frac{\partial \bar{f}}{\partial s}+\bar{g} \frac{\partial f}{\partial r}-g \frac{\partial \bar{f}}{\partial r}+D_{x}[\bar{f}] \frac{\partial f}{\partial s_{x}}-D_{x}[f] \frac{\partial \bar{f}}{\partial s_{x}} \\
& \tilde{\mathfrak{g}}=[[\sigma, \bar{\sigma}]]_{2}=\bar{f} \frac{\partial g}{\partial s}-f \frac{\partial \bar{g}}{\partial s}+\bar{g} \frac{\partial g}{\partial r}-g \frac{\partial \bar{g}}{\partial r}+D_{x}[\bar{g}] \frac{\partial g}{\partial r_{x}}-D_{x}[g] \frac{\partial \bar{g}}{\partial r_{x}} .
\end{aligned}
$$

THEOREM 2.8 (see [34]). The commutator of the hydrodynamic symmetries (2.22) and (2.12) with the characteristics $\bar{\sigma}$ and $\sigma$ is again a hydrodynamic symmetry of system (2.21) with the characteristic $\tilde{\sigma}=[[\sigma, \bar{\sigma}]]=(\tilde{f}, \tilde{g})$ generated by the Lie equations

$$
s_{\tilde{\tau}}=\tilde{f}=\tilde{\bar{\phi}}(x, t, s, r) s_{x}+\tilde{\bar{b}}(s), \quad r_{\tilde{\tau}}=\tilde{g}=\tilde{\bar{\psi}}(x, t, s, r) r_{x}+\tilde{\bar{d}}(r),
$$

with the coefficients $\tilde{\bar{\phi}}, \tilde{\bar{\psi}}$ determined by (2.13) or (2.14) for $\beta \neq 0$ or for $\beta=0$, respectively, and the change of notation for any value of $\beta$ :

$$
\begin{aligned}
& (a(s, r), c(s, r)) \longmapsto(\tilde{\bar{a}}(s, r), \tilde{\bar{c}}(s, r)), \\
& (\hat{\Phi}(s, r, t), \hat{\Theta}(s, r, t)) \longmapsto(\hat{\tilde{\Phi}}(s, r, t), \hat{\tilde{\Theta}}(s, r, t)) \text {, } \\
& (\phi(s, r, t), \psi(s, r, t)) \longmapsto(\hat{\tilde{\phi}}(s, r, t), \hat{\tilde{\psi}}(s, r, t)), \\
& \tilde{\bar{b}}(s)=\left|\begin{array}{cc}
\bar{b}(s) & b(s) \\
\overline{b^{\prime}}(s) & b^{\prime}(s)
\end{array}\right|, \quad \tilde{\bar{d}}(r)=\left|\begin{array}{cc}
\bar{d}(r) & d(r) \\
\overline{d^{\prime}}(r) & d^{\prime}(r)
\end{array}\right| .
\end{aligned}
$$

In Theorem 2.8 the following notation is used:

$$
\begin{aligned}
\tilde{\tilde{\phi}}(s, r, t)= & \tilde{\bar{b}}(s) \phi_{s}(s, r, t)+\tilde{\bar{d}}(r) \phi_{r}(s, r, t), \\
\tilde{\tilde{\psi}}(s, r, t)= & \tilde{\bar{b}}(s) \psi_{s}(s, r, t)+\tilde{\bar{d}}(r) \psi_{r}(s, r, t), \\
\hat{\tilde{\Phi}}(s, r, t)= & \bar{b}(s) \hat{\Phi}_{s}+\bar{d}(r) \hat{\Phi}_{r}-b(s) \hat{\bar{\Phi}}_{s}-d(r) \hat{\bar{\Phi}}_{r}, \\
\tilde{\tilde{\Theta}}(s, r, t)= & \bar{b}(s) \hat{\Theta}_{s}+\bar{d}(r) \hat{\Theta}_{r}-b(s) \hat{\bar{\Theta}}_{s}-d(r) \hat{\bar{\Theta}}_{r}, \\
\tilde{\bar{a}}(s, r)= & \bar{b}(s)\left[a_{s}(s, r)-\Phi_{s} a(s, r)\right]-b(s)\left[\bar{a}_{s}(s, r)-\Phi_{s} \bar{a}(s, r)\right] \\
& +\Phi_{r}[d(r) \bar{c}(s, r)-\bar{d}(r) c(s, r)]+\Phi_{0}(s) \bar{a}(s, r)-\bar{\Phi}_{0}(s) a(s, r), \\
\tilde{\bar{c}}(s, r)= & \bar{d}(r)\left[c_{r}(s, r)-\Theta_{r} c(s, r)\right]-d(r)\left[\bar{c}_{r}(s, r)-\Theta_{r} \bar{c}(s, r)\right] \\
& +\Theta_{s}[b(s) \bar{a}(s, r)-\bar{b}(s) a(s, r)]+\Theta_{0}(r) \bar{c}(s, r)-\bar{\Theta}_{0}(r) c(s, r),
\end{aligned}
$$

with $\Phi=\Phi(s, r, 0)$ and $\Theta=\Theta(s, r, 0)$ if $\beta \neq 0$, whereas $\Phi=\Phi(s, r)$ and $\Theta=\Theta(s, r)$ if $\beta=0$. 
COROLLARY 2.9. Formulas (2.30) determine recursions of solutions for the linear systems (2.11) and (2.15), respectively. They map any pair of solutions of the corresponding system again into its solution.

Consider a special case $b(s)=d(r)=0, \hat{\Phi}=\hat{\Theta}=c_{0}=0,(\bar{b}(s), \bar{d}(r)) \neq(0,0)$.

COROLLARY 2.10. Let condition (1) of Theorem 2.2 be satisfied for system (2.1) and let the hydrodynamic symmetry inhomogeneous in derivatives of the form (2.22) exist. Then the Lie commutator of symmetry (2.22) and the hydrodynamic symmetry homogeneous in derivatives of the form (2.18), (2.19), and (2.20) with $c_{0}=0$ is again a homogeneous symmetry of the same form:

$$
s_{\tilde{\tau}}=\tilde{f}=\tilde{\bar{\phi}}(x, t, s, r) s_{x}, \quad r_{\tilde{\tau}}=\tilde{g}=\tilde{\bar{\psi}}(x, t, s, r) r_{x},
$$

where for any real value of $\beta$,

$$
\begin{aligned}
& \tilde{\bar{\phi}}(x, t, s, r)= \tilde{\bar{a}}(s, r) \exp \left\{\beta\left[x+\int_{0}^{t} \phi(s, r, t) d t\right]\right\}, \\
& \tilde{\bar{\psi}}(x, t, s, r)=\tilde{\bar{c}}(s, r) \exp \left\{\beta\left[x+\int_{0}^{t} \psi(s, r, t) d t\right]\right\}, \\
& \tilde{\bar{a}}(s, r)= \bar{b}(s) a_{s}(s, r)-\left[\bar{b}(s) \Phi_{s}(s, r, 0)+\bar{\Phi}_{0}(s)\right] a(s, r) \\
&-\bar{d}(r) \Phi_{r}(s, r, 0) c(s, r), \\
& \tilde{\bar{c}}(s, r)= \bar{d}(r) c_{r}(s, r)-\left[\bar{d}(r) \Theta_{r}(s, r, 0)+\bar{\Theta}_{0}(r)\right] c(s, r) \\
&-\bar{b}(s) \Theta_{s}(s, r, 0) a(s, r) .
\end{aligned}
$$

Thus, according to Corollary 2.10, the Lie commutator with the inhomogeneous-inderivatives symmetry (2.22) is a linear operator acting on the space of homogeneous hydrodynamic symmetries with $c_{0}=0$. It generates recursion (2.33) of solutions for the linear system (2.15).

REMARK 2.11. The hydrodynamic symmetry inhomogeneous in derivatives generates by the Lie commutator a recursion operator for homogeneous hydrodynamic symmetries.

COROLlary 2.12. If system (2.1) satisfies condition (1) of Theorem 2.2, then it admits an infinite set of mutually commuting hydrodynamic symmetries homogeneous in derivatives of the form (2.31) and (2.32) with $c_{0}=0$ depending on arbitrary functions.

In particular, if for $\beta=0$, the coefficients $\phi(s, r)$ and $\psi(s, r)$ of (2.1) do not depend explicitly upon $t$, then we reproduce Tsarëv's result [39] about the commutativity of flows of the hydrodynamic type with no explicit dependence on $t, x$.

2.3. Hydrodynamic symmetries of diagonal systems with an explicit space dependence. Consider a diagonal system of the hydrodynamic type with an explicit dependence on $x$ :

$$
s_{t}=\phi^{*}(s, r, x) s_{x}, \quad r_{t}=\psi^{*}(s, r, x) r_{x} .
$$


Define the functions $\Phi(s, r, x)$ and $\Theta(s, r, x)$ by the following equations:

$$
\begin{aligned}
& \Phi_{r}(s, r, x)=\frac{\left[\phi^{*-1}(s, r, x)\right]_{r}}{\left[\phi^{*-1}(s, r, x)-\psi^{*^{-1}}(s, r, x)\right]}, \\
& \Theta_{s}(s, r, x)=\frac{\left[\psi^{*-1}(s, r, x)\right]_{s}}{\left[\psi^{*-1}(s, r, x)-\phi^{*^{-1}}(s, r, x)\right]} .
\end{aligned}
$$

THEOREM 2.13. A diagonal generic system of the hydrodynamic type (2.34) which may explicitly depend on a space variable $x$ admits an infinite set of hydrodynamic symmetries with a functional arbitrariness locally parameterized by the two arbitrary functions of one variable $c_{1}(s)$ and $c_{2}(r)$ if and only if the following two conditions are satisfied:

(1) coefficients $\phi^{*}, \psi^{*}$ of system (2.34) satisfy the equations

$$
\Phi_{r x}(s, r, x)=\beta\left(\phi^{*-1}(s, r, x)\right)_{r}, \quad \Theta_{s x}(s, r, x)=\beta\left(\psi^{*-1}(s, r, x)\right)_{s},
$$

with an arbitrary real constant $\beta$;

(2) there exist four functions of one variable $b(s), d(r), \Phi_{0}(s)$, and $\Theta_{0}(r)$ satisfying (2.11), where the functions $\hat{\Phi}(s, r, x)$ and $\hat{\Theta}(s, r, x)$ are defined by (2.7) with $t$ changed to $x$. These symmetries are generated by the Lie equations

$$
s_{\tau}=\tilde{\phi}^{*}(x, t, s, r) s_{x}+b(s), \quad r_{\tau}=\tilde{\psi}^{*}(x, t, s, r) r_{x}+d(r)
$$

The functions $\tilde{\phi}^{*}, \tilde{\psi}^{*}$ are defined for $\beta \neq 0$ and $\beta=0$, respectively, as

$$
\begin{aligned}
& \begin{array}{l}
\tilde{\phi}^{*}(x, t, s, r) \\
\quad=\phi^{*}(s, r, x)\left\{a(s, r) \exp \left[\beta\left(t+\int_{0}^{x} \phi^{*-1}(s, r, x) d x\right)\right]+\frac{1}{\beta} \hat{\Phi}(s, r, x)\right\}, \\
\tilde{\psi}^{*}(x, t, s, r) \\
\quad=\psi^{*}(s, r, x)\left\{c(s, r) \exp \left[\beta\left(t+\int_{0}^{x} \psi^{*-1}(s, r, x) d x\right)\right]+\frac{1}{\beta} \hat{\Theta}(s, r, x)\right\}, \\
\tilde{\phi}^{*}(x, t, s, r) \\
\quad=\phi^{*} \cdot\left\{a(s, r)+\int_{0}^{x} \hat{\phi}(s, r, x) d x-\hat{\Phi}(s, r)\left[t+\int_{0}^{x} \phi^{*-1}(s, r, x) d x\right]\right\}, \\
\tilde{\psi}^{*}(x, t, s, r) \\
\quad=\psi^{*} \cdot\left\{c(s, r)+\int_{0}^{x} \hat{\psi}(s, r, x) d x-\hat{\Theta}(s, r)\left[t+\int_{0}^{x} \psi^{*-1}(s, r, x) d x\right]\right\} .
\end{array}
\end{aligned}
$$

Here the functions $\hat{\phi}, \hat{\psi}$ are defined as follows:

$$
\begin{aligned}
& \hat{\phi}(s, r, x)=b(s)\left[\phi^{*-1}(s, r, x)\right]_{s}+d(r)\left[\phi^{*-1}(s, r, x)\right]_{r}, \\
& \hat{\psi}(s, r, x)=b(s)\left[\psi^{*-1}(s, r, x)\right]_{s}+d(r)\left[\psi^{*^{-1}}(s, r, x)\right]_{r} .
\end{aligned}
$$

The functions $a(s, r)$ and $c(s, r)$ form an arbitrary solution of the linear system (2.15) with the coefficients $\Phi_{r}(s, r, 0)$ and $\Theta_{s}(s, r, 0)$ obtained from (2.35) at $x=0$. 
2.4. Invariant solutions and linearizing transformations for the systems with an explicit dependence on $t$ or $x$. Let system (2.1) which may explicitly depend on $t$ satisfy condition (1) of Theorem 2.2 and hence admit an infinite set of hydrodynamic symmetries homogeneous in derivatives (2.18). For the corresponding invariant solutions, we have $s_{\tau}=r_{\tau}=0$ which implies that $\tilde{\phi}(x, t, s, r)=0$ and $\tilde{\psi}(x, t, s, r)=0$ due to the condition $s_{x} r_{x} \neq 0$. Using here formulas (2.19) and (2.20) for $\tilde{\phi}, \tilde{\psi}$ with $c_{0}=1$, we obtain the following conditions determining the invariant solutions for $\beta \neq 0$ and $\beta=0$, respectively:

$$
\begin{gathered}
a(s, r)+\exp \left\{-\beta\left[x+\int_{0}^{t} \phi(s, r, t) d t\right]\right\}=0, \\
c(s, r)+\exp \left\{-\beta\left[x+\int_{0}^{t} \psi(s, r, t) d t\right]\right\}=0, \\
a(s, r)+x+\int_{0}^{t} \phi(s, r, t) d t=0, \quad c(s, r)+x+\int_{0}^{t} \psi(s, r, t) d t=0 .
\end{gathered}
$$

TheOREM 2.14 (see [34]). Let the coefficients $\phi, \psi$ of diagonal system (2.1) satisfy condition (1) of Theorem 2.2. Then any solution of system (2.40) for $\beta \neq 0$ or of system (2.41) for $\beta=0$ is also a solution of system (2.1) if the following conditions are satisfied for $\beta \neq 0$ and $\beta=0$, respectively:

$$
\begin{gathered}
\left\{\left[\ln |a(s, r)|+\beta \int_{0}^{t} \phi(s, r, t) d t\right]_{s}\right. \\
\left.\cdot\left[\ln |c(s, r)|+\beta \int_{0}^{t} \psi(s, r, t) d t\right]_{r}\right\}\left.\right|_{(2.40)} \neq 0, \\
\left.\left\{\left[a(s, r)+\int_{0}^{t} \phi(s, r, t) d t\right]_{s} \cdot\left[c(s, r)+\int_{0}^{t} \psi(s, r, t) d t\right]_{r}\right\}\right|_{(2.41)} \neq 0 .
\end{gathered}
$$

Vice versa, any smooth solution $s(x, t), r(x, t)$ of system (2.1) can be obtained from systems (2.40) or (2.41) if condition (2.42) is satisfied in the vicinity of any point $\left(x_{0}, t_{0}\right)$, where $s_{x}\left(x_{0}, t_{0}\right) \cdot r_{x}\left(x_{0}, t_{0}\right) \neq 0$.

REMARK 2.15 (see [34]). Equations (2.40) for $\beta \neq 0$ and (2.41) for $\beta=0$ determine an implicit form of the linearizing point transformation for the nonlinear system (2.1) with an explicit $t$-dependence satisfying condition (1) of Theorem 2.2. Indeed, the search for solutions $s(x, t), r(x, t)$ of the nonlinear system (2.1) is now reduced to the search for solutions $a(s, r), c(s, r)$ of the linear system (2.15) with variable coefficients. In particular, if the coefficients $\phi(s, r)$ and $\psi(s, r)$ of system (2.1) have no explicit dependence on $t$, then condition (1) of Theorem 2.2 is satisfied with $\beta=0$ and (2.41) coincide with the classical hodograph transformation [27]: $a(s, r)+x+t \phi(s, r)=0$ and $c(s, r)+x+t \psi(s, r)=0$.

Consider now system (2.34) with an explicit $x$-dependence. Let it satisfy condition (2.36) of Theorem 2.13 in order to admit an infinite set of the hydrodynamic symmetries (2.37) with $b(s)=d(r)=0$. Then for invariant solutions determined by the equations 
$s_{T}=r_{T}=0$ with the condition $s_{x} r_{x} \neq 0$, we obtain $\tilde{\phi}^{*}(x, t, s, r)=0$ and $\tilde{\psi}^{*}(x, t, s, r)=$ 0 . Here the functions $\tilde{\phi}^{*}, \tilde{\psi}^{*}$ are defined by (2.38) for $\beta \neq 0$ and $\beta=0$ with $\hat{\Phi}=\Phi_{0}(s)$, $\hat{\Theta}=\Theta_{0}(r)$, and $\hat{\phi}=\hat{\psi}=0$. These equations determine a linearizing transformation for system (2.34) with an explicit dependence on $x$ if the existence conditions for the implicit vector function similar to (2.42) are satisfied.

2.5. Higher symmetries of diagonal two-component systems. Higher symmetries of the second order are generated by the Lie equations (2.3) with $N=2$ :

$$
s_{\tau}=f\left(x, t, s, r, s_{x}, r_{x}, s_{x x}, r_{x x}\right), \quad r_{\tau}=g\left(x, t, s, r, s_{x}, r_{x}, s_{x x}, r_{x x}\right)
$$

which are compatible with system (2.1).

DEFINITION 2.16. System (2.1) is called generic with respect to the second-order symmetries if its coefficients $\phi, \psi$ do not satisfy the constraints

$$
\begin{aligned}
\Phi(s, r, t) & =\ln \left|\frac{\phi_{s}}{C(s, t) \phi+D(s, t)}\right|, & \Theta(s, r, t) & =\ln \left|\frac{\psi_{r}}{G(r, t) \psi+H(r, t)}\right|, \\
\Phi_{t}(s, r, t) & =A(s, t) \phi+B(s, t), & \Theta_{t}(s, r, t) & =E(r, t) \psi+F(r, t),
\end{aligned}
$$

with arbitrary functions $A, B, C, D, E, F, G, H$ and $\Phi, \Theta$ defined by (2.6).

For the symmetries (2.43) of the generic system (2.1), the following results hold.

THEOREM 2.17. The necessary existence condition for the second-order symmetries of the generic system (2.1) coincides with condition (1) of Theorem 2.2, necessary and sufficient for system (2.1) to admit an infinite set of hydrodynamic symmetries homogeneous in derivatives.

Define the functions $\Lambda, \hat{\Phi}$, and $\hat{\Theta}$ by the following equations:

$$
\begin{aligned}
\Lambda_{s r}(s, r)= & -\Phi_{r}(s, r, t) \Theta_{s}(s, r, t), \\
\hat{\Phi}(s, r, t)= & A(s)\left(\Phi_{s}^{2}-\Phi_{s s}+2 \Lambda_{s s}\right)+A^{\prime}(s) \Lambda_{s}+C(r)\left(2 \Phi_{r} \Theta_{r}+\Phi_{r r}-\Phi_{r}^{2}\right) \\
& +C^{\prime}(r) \Phi_{r}+b(s) \Phi_{s}+d(r) \Phi_{r}+\Phi_{0}(s), \\
\hat{\Theta}(s, r, t)= & A^{\prime}(s) \Theta_{s}+A(s)\left(2 \Theta_{s} \Phi_{s}+\Theta_{s s}-\Theta_{s}^{2}\right)+C(r)\left(\Theta_{r}^{2}-\Theta_{r r}+2 \Lambda_{r r}\right) \\
& +C^{\prime}(r) \Lambda_{r}(s, r)+b(s) \Theta_{s}+d(r) \Theta_{r}+\Theta_{0}(r),
\end{aligned}
$$

where $A(s), C(r), b(s), d(r), \Phi_{0}(s)$, and $\Theta_{0}(r)$ are arbitrary smooth functions of one variable and the function $\Lambda(s, r)$ has no explicit dependence on $t$ as a consequence of the first equation of (2.45) and (2.6), (2.10). If $\beta=0$ in (2.10), then in virtue of condition (2.16) the functions $\Phi(s, r), \Theta(s, r)$ do not depend explicitly on $t$. Then define the functions $\hat{\phi}, \hat{\psi}$ :

$$
\begin{aligned}
\hat{\phi}(s, r, t)= & A(s)\left(2 \Phi_{s} \phi_{s}-\phi_{s s}\right)+C(r)\left[2 \Theta_{r} \phi_{r}+\phi_{r r}-2 \Phi_{r}\left(\phi_{r}-\psi_{r}\right)\right] \\
& +C^{\prime}(r) \phi_{r}+b(s) \phi_{s}+d(r) \phi_{r} \\
\hat{\psi}(s, r, t)= & A(s)\left[2 \Phi_{s} \psi_{s}+\psi_{s s}-2 \Theta_{s}\left(\psi_{s}-\phi_{s}\right)\right]+A^{\prime}(s) \psi_{s} \\
& +C(r)\left(2 \Theta_{r} \psi_{r}-\psi_{r r}\right)+b(s) \psi_{s}+d(r) \psi_{r} .
\end{aligned}
$$


THEOREM 2.18 (see [36]). The diagonal two-component generic hydrodynamic-type system (2.1) which may explicitly depend on $t$ admits an infinite set of second-order symmetries with a functional arbitrariness which is locally parameterized by two arbitrary functions of one variable $c_{1}(s)$ and $c_{2}(r)$ if and only if the following two conditions are satisfied:

(1) the coefficients $\phi, \psi$ of system (2.1) satisfy (2.10) with an arbitrary real constant $\beta$, where the functions $\Phi, \Theta$ are defined by (2.6);

(2) there exist six functions $A(s), C(r), b(s), d(r), \Phi_{0}(s)$, and $\Theta_{0}(r)$ of one variable satisfying (2.11) with $\hat{\Phi}(s, r, t)$ and $\hat{\Theta}(s, r, t)$ defined by (2.45).

The symmetries in Theorem 2.18 are generated by the Lie equations

$$
\begin{aligned}
& s_{\tau}=f=A(s) \frac{s_{x x}}{s_{x}^{2}}+\Phi_{r}\left[A(s) \frac{r_{x}}{s_{x}}+C(r) \frac{s_{x}}{r_{x}}\right]+\beta \frac{A(s)}{s_{x}}+s_{x} v+2 A(s) \Phi_{s}+b(s), \\
& r_{\tau}=g=C(r) \frac{r_{x x}}{r_{x}^{2}}+\Theta_{s}\left[A(s) \frac{r_{x}}{s_{x}}+C(r) \frac{s_{x}}{r_{x}}\right]+\beta \frac{C(r)}{r_{x}}+r_{x} \rho+2 C(r) \Theta_{r}+d(r),
\end{aligned}
$$

where the coefficients $v(x, t, s, r)$ and $\rho(x, t, s, r)$ are defined by formulas (2.13) and (2.14) for $\beta \neq 0$ and $\beta=0$, respectively, with the change of $\tilde{\phi}$ to $v$ and $\tilde{\psi}$ to $\rho$, where $\hat{\phi}, \hat{\psi}$ are defined by formulas (2.46). The functions $a(s, r)$ and $c(s, r)$ form an arbitrary smooth solution of the linear system (2.15).

REMARK 2.19. For $A(s)=C(r)=0$, the higher symmetries (2.47) reduce to the hydrodynamic symmetries (2.12), and the coefficients $v, \rho$ coincide with $\tilde{\phi}, \tilde{\psi}$.

2.6. First-order recursion operators. An effective description of an infinite set of symmetries of any order is obtained by means of recursion operators which, by their definition, map any symmetry again into a symmetry. Here we consider recursion operators from the class of matrix differential operators:

$$
R=A_{N} D_{x}^{N}+A_{N-1} D_{x}^{N-1}+\cdots+A_{1} D_{x}+A_{0} .
$$

For $n$-component systems, $A_{i}$ are $n \times n$ matrices. We set $n=2$ until Section $5 . A_{i}$ may depend on $s, r$ and their derivatives of finite orders with respect to $x$.

DEFINITION 2.20. If $A_{N} \neq 0$, then $N$ is called the order of the recursion operator (2.48). Consider the case $N=1$. Define the functions

$$
\begin{aligned}
& S(s, r)=\hat{\Phi}(s, r, 0)=A(s) \Phi_{s}(s, r, 0)+C(r) \Phi_{r}(s, r, 0)+\Phi_{0}(s), \\
& T(s, r)=\hat{\Theta}(s, r, 0)=A(s) \Theta_{s}(s, r, 0)+C(r) \Theta_{r}(s, r, 0)+\Theta_{0}(r),
\end{aligned}
$$

where $A(s), C(r), \Phi_{0}(s)$, and $\Theta_{0}(r)$ are arbitrary smooth functions of one variable.

THEOREM 2.21 (see [36]). Let system (2.1) satisfy condition (1) of Theorem 2.2. Then a first-order recursion operator for system (2.1) exists if and only if there exist such functions $A(s)$ and $C(r)$ which satisfy the conditions

$$
S_{r}(s, r)=\Phi_{r}(s, r, 0)(S-T), \quad T_{S}(s, r)=\Theta_{s}(s, r, 0)(T-S),
$$


with $S(s, r)$ and $T(s, r)$ defined by (2.49), where $\Phi_{0}(s)=\Theta_{0}(r)=0$ (this is equivalent to a redefinition of $\Phi_{s}(s, r, 0)$ and $\left.\Theta_{r}(s, r, 0)\right)$. This recursion operator has the form

$$
\begin{aligned}
R=\{ & \left(\left(\begin{array}{cc}
A(s) & 0 \\
0 & C(r)
\end{array}\right)\right) \cdot\left(D_{x}-\beta\right) \\
& -\left(\left(\begin{array}{cc}
A(s) D_{x}[\Phi(s, r, t)] & -\Phi_{r}(s, r, t)\left[A(s) r_{x}-C(r) s_{x}\right] \\
\Theta_{s}(s, r, t)\left[A(s) r_{x}-C(r) s_{x}\right] & C(r) D_{x}[\Theta(s, r, t)]
\end{array}\right)\right\} \\
& \times\left(\left(\begin{array}{cc}
\frac{1}{s_{x}} & 0 \\
0 & \frac{1}{r_{x}}
\end{array}\right)\right) .
\end{aligned}
$$

The first example of first-order recursion operators for a class of two-component systems of the hydrodynamic type was given in [30]. Later, Teshukov [38] generalized it for $n \times n$ systems with no explicit dependence on $t$.

THEOREM 2.22. System (2.1) satisfying condition (1) of Theorem 2.2 admits firstorder recursion operator $R$ if and only if there exists a recursion operator $\hat{R}$ which acts on a subspace of hydrodynamic symmetries homogeneous in derivatives, of the form (2.18), (2.19), and (2.20) with $C_{0}=0$.

The action of $\hat{R}$ is defined by the appropriate restriction of $R$

$$
R\left(\begin{array}{l}
a(s, r) \exp \left\{\beta\left[x+\int_{0}^{t} \phi(s, r, t) d t\right]\right\} s_{x} \\
c(s, r) \exp \left\{\beta\left[x+\int_{0}^{t} \psi(s, r, t) d t\right]\right\} r_{x}
\end{array}\right)=\left(\begin{array}{l}
\hat{a}(s, r) \exp \left\{\beta\left[x+\int_{0}^{t} \phi(s, r, t) d t\right]\right\} s_{x} \\
\hat{c}(s, r) \exp \left\{\beta\left[x+\int_{0}^{t} \psi(s, r, t) d t\right]\right\} r_{x}
\end{array}\right)
$$

with the functions $\hat{a}(s, r)$ and $\hat{c}(s, r)$ defined by the formulas

$$
\begin{aligned}
\hat{a}(s, r)= & A(s)\left[a_{s}(s, r)-\Phi_{s}(s, r, 0) a(s, r)\right]-\Phi_{0}(s) a(s, r) \\
& -C(r) \Phi_{r}(s, r, 0) c(s, r), \\
\hat{c}(s, r)= & C(r)\left[c_{r}(s, r)-\Theta_{r}(s, r, 0) c(s, r)\right]-\Theta_{0}(r) c(s, r) \\
& -A(s) \Theta_{s}(s, r, 0) a(s, r) .
\end{aligned}
$$

COROLlARY 2.23. For any smooth solution $a(s, r), c(s, r)$ of the linear system (2.15), the functions $\hat{a}(s, r)$ and $\hat{c}(s, r)$ defined by (2.53) also form a solution of this system if and only if condition (2.50) is satisfied. Therefore, the formulas (2.53) determine a recursion of solutions of system (2.15).

REMARK 2.24. In virtue of the linearizing transformations (2.40) or (2.41), a search for any "nonsingular" solutions of the nonlinear system (2.1) reduces to a search for solutions $a(s, r), c(s, r)$ of the linear system (2.15) with variable coefficients. However, the integration of (2.15) may also be a problem. The existence of a recursion operator and hence of the recursion (2.53) which generates new solutions of the linear equations (2.15) out of known "seed" solutions is the important property of system (2.1). It allows 
us to pass from a linearization of system (2.1) to its integration if the coefficients $\phi, \psi$ satisfy condition (1) of Theorem 2.2 and conditions (2.50).

REMARK 2.25. Linear system (2.15) has two trivial solutions $(a, c)=(-1,-1)$ and $(a(s, r), c(s, r))=(\phi(s, r, 0), \psi(s, r, 0))$ (see (2.6)). Recursion formulas (2.53) map them to nontrivial solutions $\hat{a}(s, r), \hat{c}(s, r)$ of (2.15).

In particular, the first solution is mapped to the solution (2.49): $\hat{a}=S(s, r)$ and $\hat{c}=$ $T(s, r)$. Substituting these new solutions for $a, c$ to (2.53), we obtain new nontrivial solutions of system (2.15). Thus, we construct two infinite series of its solutions. Then the linearizing transformations (2.40) and (2.41) generate two infinite series of exact solutions of (2.1) in an explicit form.

COROLLARY 2.26. Transformation (2.53) maps any smooth solution ( $a, c)$ of system (2.15) again into a solution $(\hat{a}, \hat{c})$ of this system if and only if it maps the trivial solution $a=1, c=1$ into some solution of (2.15).

COROLLARY 2.27. Recursion (2.33) for solutions of the linear system (2.15) generated by the Lie commutator with the hydrodynamic symmetry inhomogeneous in derivatives coincides with recursion (2.53) generated by the first-order recursion operator (2.51). The existence conditions for the recursion operator and for the hydrodynamic symmetry inhomogeneous in derivatives of system (2.1) coincide. If there exist several such symmetries of the form (2.22), then there exist several corresponding recursion operators of the form (2.51) with $A(s)=\bar{b}(s)$ and $C(r)=\bar{d}(r)$.

Recursion operator $R$ of the form (2.51) generates an infinite set of higher symmetries (2.3) of any order $N$. Second-order symmetries are generated by a two-fold action of the operator $R$ on hydrodynamic symmetries homogeneous in derivatives (2.18), (2.19), and (2.20) with $c_{0} \neq 0$, where we set $c_{0}=1$ and $a(s, r)=c(s, r)=0$. In the case $\beta=0$ according to formulas (2.18) and (2.20), this seed symmetry has the form $s_{\tau}=s_{x}[x+$ $\left.\int_{0}^{t} \phi(s, r, t) d t\right], r_{\tau}=r_{x}\left[x+\int_{0}^{t} \psi(s, r, t) d t\right]$.

\subsection{Second-order recursion operators and higher symmetries}

DEFINITION 2.28. System (2.1) is called generic with respect to second-order recursion operators if its coefficients do not satisfy any of the constraints $\Phi_{r s} / \Phi_{r}+\Theta_{s}=$ $c_{1}(s) e^{\Phi}$ or $\Theta_{s r} / \Theta_{s}+\Phi_{r}=c_{2}(r) e^{\Theta}$ with arbitrary functions $c_{1}(s)$ and $c_{2}(r)$.

Define the functions

$$
\begin{aligned}
S(s, r)= & A(s)\left(\Phi_{s}^{2}-\Phi_{s s}+2 \Lambda_{s s}\right)+A^{\prime}(s) \Lambda_{s}+C(r)\left(2 \Phi_{r} \Theta_{r}+\Phi_{r r}-\Phi_{r}^{2}\right) \\
& +C^{\prime}(r) \Phi_{r}+b(s) \Phi_{s}+d(r) \Phi_{r}+\Phi_{0}(s), \\
T(s, r)= & A^{\prime}(s) \Theta_{s}+A(s)\left(2 \Theta_{s} \Phi_{s}+\Theta_{s s}-\Theta_{s}^{2}\right)+C(r)\left(\Theta_{r}^{2}-\Theta_{r r}+2 \Lambda_{r r}\right) \\
& +C^{\prime}(r) \Lambda_{r}+b(s) \Theta_{s}+d(r) \Theta_{r}+\Theta_{0}(r),
\end{aligned}
$$

where $A(s), C(r), b(s), d(r), \Phi_{0}(s)$, and $\Theta_{0}(r)$ are arbitrary smooth functions and the functions $\Phi(s, r, t), \Theta(s, r, t)$, and $\Lambda(s, r)$ are defined by formulas (2.6) and (2.45).

THEOREM 2.29 (see [36]). Let the generic system (2.1) satisfy condition (1) of Theorem 2.2. Then second-order recursion operator of the form (2.48) with $N=2$ exists for system 
(2.1) if and only if there exist six functions $A(s), C(r), b(s), d(r), \Phi_{0}(s)$, and $\Theta_{0}(r)$ of one variable which satisfy conditions (2.50) with $S$ and $T$ defined by (2.54).

The recursion operator in Theorem 2.29 is determined by the formula

$$
R=\left(A D_{x}^{2}+B D_{x}+F\right)\left(\begin{array}{cc}
\frac{1}{s_{x}} & 0 \\
0 & \frac{1}{r_{x}}
\end{array}\right)
$$

with $2 \times 2$ matrices $A, B$ defined as follows:

$$
\begin{aligned}
A= & \left(\begin{array}{cc}
\frac{A(s)}{s_{x}} & 0 \\
0 & \frac{C(r)}{r_{x}}
\end{array}\right), \\
B=( & -\left[A(s) \frac{s_{x x}}{s_{x}^{2}}+2 A(s)\left(\Phi_{s}+\Phi_{r} \frac{r_{x}}{s_{x}}+\frac{\beta}{s_{x}}\right)+b(s)\right], \\
& -\Theta_{s}(s, r, t)\left[A(s) \frac{r_{x}}{s_{x}}-C(r) \frac{s_{x}}{r_{x}}\right], \Phi_{r}(s, r, t)\left[A(s) \frac{r_{x}}{s_{x}}-C(r) \frac{s_{x}}{r_{x}}\right] \\
& \left.-\left[C(r) \frac{r_{x x}}{r_{x}^{2}}+2 C(r)\left(\Theta_{s} \frac{s_{x}}{r_{x}}+\Theta_{r}+\frac{\beta}{r_{x}}\right)+d(r)\right]\right),
\end{aligned}
$$

and the elements $f_{i j}$ of the matrix $F$ are defined by the equations

$$
\begin{aligned}
f_{12}= & A(s)\left[-\Phi_{r} \frac{r_{x}}{s_{x}}\left(\frac{s_{x x}}{s_{x}}-\frac{r_{x x}}{r_{x}}\right)+\left(\Phi_{r r}-\Phi_{r}^{2}\right) \frac{r_{x}^{2}}{s_{x}}\right]-C(r) \Lambda_{s r} \frac{s_{x}^{2}}{r_{x}} \\
& +\left\{A(s)\left[2\left(\Phi_{r s}-\Phi_{r} \Phi_{s}\right)-\Lambda_{s r}\right]-b(s) \Phi_{r}\right\} r_{x} \\
& +\left\{C(r)\left(2 \Phi_{r} \Theta_{r}+\Phi_{r r}-\Phi_{r}^{2}\right)+\left[C^{\prime}(r)+d(r)\right] \Phi_{r}\right\} s_{x} \\
& -\beta \Phi_{r}\left[A(s) \frac{r_{x}}{s_{x}}-C(r) \frac{s_{x}}{r_{x}}\right], \\
f_{21}= & C(r)\left[\Theta_{s} \frac{s_{x}}{r_{x}}\left(\frac{s_{x x}}{s_{x}}-\frac{r_{x x}}{r_{x}}\right)+\left(\Theta_{s s}-\Theta_{s}^{2}\right) \frac{s_{x}^{2}}{r_{x}}\right]-A(s) \Lambda_{s r} \frac{r_{x}^{2}}{s_{x}} \\
& +\left\{C(r)\left[2\left(\Theta_{s r}-\Theta_{s} \Theta_{r}\right)-\Lambda_{s r}\right]-d(r) \Theta_{s}\right\} s_{x} \\
& +\left\{A(s)\left(2 \Theta_{s} \Phi_{s}+\Theta_{s s}-\Theta_{s}^{2}\right)+\left[A^{\prime}(s)+b(s)\right] \Theta_{s}\right\} r_{x} \\
& +\beta \Theta_{s}\left[A(s) \frac{r_{x}}{s_{x}}-C(r) \frac{s_{x}}{r_{x}}\right], \\
f_{11}+f_{12}= & \hat{\Phi}(s, r, t) s_{x}+\beta\left\{A(s)\left[\frac{s_{x x}}{s_{x}^{2}}+2 \Phi_{s}(s, r, t)+\frac{\beta}{s_{x}}\right]\right. \\
& \left.+\Phi_{r}(s, r, t)\left[A(s) \frac{r_{x}}{s_{x}}+C(r) \frac{s_{x}}{r_{x}}\right]+b(s)\right\}, \\
f_{21}+f_{22}= & \hat{\Theta}(s, r, t) r_{x}+\beta\left\{C(r)\left[\frac{r_{x x}}{r_{x}^{2}}+2 \Theta_{r}(s, r, t)+\frac{\beta}{r_{x}}\right]\right. \\
& \left.+\Theta_{s}(s, r, t)\left[A(s) \frac{r_{x}}{s_{x}}+C(r) \frac{s_{x}}{r_{x}}\right]+d(r)\right\} .
\end{aligned}
$$

Here $\Phi=\Phi(s, r, t)$ and $\Theta=\Theta(s, r, t)$, and $\hat{\Phi}(s, r, t)$ and $\hat{\Theta}(s, r, t)$ are defined by (2.45). 
THEOREM 2.30. For system (2.1) satisfying condition (1) of Theorem 2.2, there exists a second-order recursion operator $R$ of the form (2.55) if and only if there exists the recursion operator $\hat{R}$ acting on the subspace of hydrodynamic symmetries homogeneous in derivatives of the form (2.18), (2.19), and (2.20) with $c_{0}=0$.

In Theorem 2.30, the action of $\hat{R}$ is defined as an appropriate restriction of $R$ by (2.52) with $\hat{a}(s, r)$ and $\hat{c}(s, r)$ defined as

$$
\begin{aligned}
\hat{a}= & A(s) a_{s s}-\left[2 A(s) \Phi_{s}+b(s)\right] a_{s}-C(r) \Phi_{r} c_{r} \\
& +\left[A^{\prime}(s) \Lambda_{s}+A(s)\left(\Phi_{s}^{2}-\Phi_{s s}+2 \Lambda_{s s}\right)+b(s) \Phi_{s}+\Phi_{0}(s)\right] a \\
& +\left[C^{\prime}(r) \Phi_{r}+C(r)\left(2 \Phi_{r} \Theta_{r}+\Phi_{r r}-\Phi_{r}^{2}\right)+d(r) \Phi_{r}\right] c, \\
\hat{c}= & C(r) c_{r r}-\left[2 C(r) \Theta_{r}+d(r)\right] c_{r}-A(s) \Theta_{s} a_{s} \\
& +\left[C^{\prime}(r) \Lambda_{r}+C(r)\left(\Theta_{r}^{2}-\Theta_{r r}+2 \Lambda_{r r}\right)+d(r) \Theta_{r}+\Theta_{0}(r)\right] c \\
& +\left[A^{\prime}(s) \Theta_{s}+A(s)\left(2 \Theta_{s} \Phi_{s}+\Theta_{s s}-\Theta_{s}^{2}\right)+b(s) \Theta_{s}\right] a .
\end{aligned}
$$

COROLlary 2.31. For any smooth solution $a(s, r), c(s, r)$ of the linear system (2.15), the functions $\hat{a}(s, r)$ and $\hat{c}(s, r)$ defined by formulas (2.58) form also a solution of this system if and only if condition (2.50) for the functions (2.54) is satisfied. Formulas (2.58) determine a recursion of solutions for system (2.15).

Remarks 2.24 and 2.25 and Corollary 2.26 are still valid for recursion (2.58). Thus, we can again construct two infinite series of solutions of system (2.1) starting from a trivial solution of system (2.15).

THEOREM 2.32. Existence conditions (2.50) with the notation (2.54) for the secondorder recursion of solutions of system (2.15) are less restrictive than the existence conditions (2.50) with the notation (2.49) for the first-order recursion (2.53).

Higher symmetries of system (2.1) are generated by the action of the recursion operator (2.55) on the hydrodynamic symmetries homogeneous in derivatives (2.18), (2.19), and (2.20) with $c_{0} \neq 0$.

Theorem 2.33 (see [36]). All the second-order symmetries (2.47) of system (2.1) obtained as a general solution of determining equations coincide with the symmetries obtained by the action of the second-order recursion operator (2.55) on the hydrodynamic symmetries (2.18), (2.19), and (2.20) with $c_{0} \neq 0$. Existence conditions for the secondorder symmetries and for the second-order recursion operator also coincide.

COROLLARY 2.34. All the second-order symmetries are obtained by an action of the second-order recursion operator on the hydrodynamic symmetries homogeneous in derivatives.

REMARK 2.35. The method of calculation of higher-order recursion operators developed in [35] is much more simple than a straightforward calculation of higher symmetries from determining equations. Thus, with the suitable extension of Corollary 2.34, we see that this easier way of calculation of symmetries by means of the Nth-order recursion operators will give all higher symmetries of the same order. Multiple application of the recursion operator of the order less than $N$ will not give the general form 
of the Nth-order symmetry. In particular, squared first-order recursion operator does not give a general form of the second-order symmetry.

\section{Generalized gas dynamics equations}

3.1. Symmetries of one-dimensional isentropic gas dynamics equations. Consider the one-dimensional gas dynamics equations for the isentropic plane-parallel gas flow:

$$
u_{t}+u u_{x}+\alpha^{2}(\rho) \rho \rho_{x}=0, \quad \rho_{t}+\rho u_{x}+u \rho_{x}=0
$$

Here $u(x, t)$ and $\rho(x, t)$ are gas velocity and density at the point $x$ at the time $t, c=$ $\rho \alpha(\rho)$ is the speed of sound, and $\alpha(\rho)$ is an arbitrary smooth function. In practice, $\alpha(\rho)$ is determined by the gas state equation $p=P(\rho)$, where $p$ is a gas pressure: $\alpha(\rho)=(1 / \rho) \sqrt{P^{\prime}(\rho)}$.

THEOREM 3.1 (see [27]). System (3.1) can be brought to the diagonal form (2.1),

$$
s_{t}=-\left[\frac{s+r}{2}-\rho \alpha(\rho)\right] s_{x}, \quad r_{t}=-\left[\frac{s+r}{2}+\rho \alpha(\rho)\right] r_{x},
$$

by the transformation from $u, \rho$ to the Riemann invariants $s=u-\int_{\rho_{0}}^{\rho} \alpha(\rho) d \rho$ and $r=u+\int_{\rho_{0}}^{\rho} \alpha(\rho) d \rho,\left(u=(r+s) / 2, \int_{\rho_{0}}^{\rho} \alpha(\rho) d \rho=(r-s) / 2\right)$, where $\rho_{0}$ is an arbitrary constant and the last equation determines $\rho$ as an implicit function $\rho=\rho(r-s)$ for any fixed choice of the function $\alpha(\rho)$.

The determining equation for symmetries of system (3.2) has the form

$$
\left(I D_{t}+A\right)(f, g)^{T}=(0,0)^{T},
$$

where $I=\left(\begin{array}{ll}1 & 0 \\ 0 & 1\end{array}\right), T$ denotes a transposed matrix, the total derivative operator $D_{t}$ is calculated with the use of (3.2), and the operator $A$ is given by

$$
A=\left(\begin{array}{cc}
\frac{s+r}{2}-\rho \alpha(\rho) & 0 \\
0 & \frac{s+r}{2}+\rho \alpha(\rho)
\end{array}\right) D_{x}+\left(\begin{array}{cc}
s_{x} & 0 \\
0 & r_{x}
\end{array}\right)\left(I+\frac{\rho \alpha^{\prime}(\rho)}{2 \alpha(\rho)}\left(\begin{array}{cc}
1 & -1 \\
-1 & 1
\end{array}\right)\right)
$$

Characteristics of the $N$ th-order symmetries (2.3) with no explicit dependence on $x, t$ were obtained for $N=1,2,3$ as general solutions of (3.3) for a generic $\alpha(\rho)$ (see [29, 30]).

CASE $N=1$.

$$
f_{1}=a(s, r) s_{x}, \quad g_{1}=c(s, r) r_{x},
$$

where $a(s, r), c(s, r)$ is an arbitrary smooth solution of the linear system

$$
a_{r}(s, r)=\frac{\alpha^{\prime}(\rho)}{4 \alpha^{2}}(a-c), \quad c_{s}(s, r)=\frac{\alpha^{\prime}(\rho)}{4 \alpha^{2}}(a-c) .
$$


CASE $N=2$.

$$
f_{2}=-\frac{s_{x x}}{s_{x}^{2}}-\frac{\alpha^{\prime}(\rho)}{4 \alpha^{2}} \frac{\left(s_{x}-r_{x}\right)^{2}}{s_{x} r_{x}}, \quad g_{2}=-\frac{r_{x x}}{r_{x}^{2}}+\frac{\alpha^{\prime}(\rho)}{4 \alpha^{2}} \frac{\left(s_{x}-r_{x}\right)^{2}}{s_{x} r_{x}} .
$$

CASE $N=3$.

$$
\begin{aligned}
f_{3}= & -\left(\frac{s_{x x x}}{s_{x}^{3}}-\frac{3 s_{x x}^{2}}{s_{x}^{4}}\right)-\frac{\alpha^{\prime}(\rho)}{4 \alpha^{2}}\left(\frac{1}{s_{x}^{3}}-\frac{1}{r_{x}^{3}}\right) s_{x} r_{x x} \\
& -\frac{3 \alpha^{\prime}(\rho)}{4 \alpha^{2}} \frac{s_{x x}}{s_{x}^{3}}\left(s_{x}-r_{x}\right)-\left[\frac{1}{2}\left(\frac{\alpha^{\prime}}{\alpha^{2}}\right)^{2}\left(\frac{1}{s_{x}}+\frac{1}{r_{x}}\right)-\left(\frac{\alpha^{\prime}}{\alpha^{2}}\right)^{\prime} \frac{1}{\alpha s_{x}}\right] \frac{\left(s_{x}-r_{x}\right)^{3}}{8 s_{x} r_{x}}, \\
g_{3}= & -\left(\frac{r_{x x x}}{r_{x}^{3}}-\frac{3 r_{x x}^{2}}{r_{x}^{4}}\right)-\frac{\alpha^{\prime}(\rho)}{4 \alpha^{2}}\left(\frac{1}{s_{x}^{3}}-\frac{1}{r_{x}^{3}}\right) r_{x} s_{x x} \\
& -\frac{3 \alpha^{\prime}(\rho)}{4 \alpha^{2}} \frac{r_{x x}}{r_{x}^{3}}\left(s_{x}-r_{x}\right)+\left[\frac{1}{2}\left(\frac{\alpha^{\prime}}{\alpha^{2}}\right)^{2}\left(\frac{1}{s_{x}}+\frac{1}{r_{x}}\right)-\left(\frac{\alpha^{\prime}}{\alpha^{2}}\right)^{\prime} \frac{1}{\alpha r_{x}}\right] \frac{\left(s_{x}-r_{x}\right)^{3}}{8 s_{x} r_{x}} .
\end{aligned}
$$

$N$ th-order symmetries are defined up to the addition of lower-order symmetries, in particular (3.5), so they have functional arbitrariness determined by the linear system (3.6).

The following special choices of $\alpha(\rho)$ lead to new second-order symmetries.

(1) The function $\alpha(\rho)$ satisfies the differential equation

$$
\left[\frac{(c \rho+b) \alpha^{2}(\rho)}{\alpha^{\prime}(\rho)}\right]_{\rho}^{\prime}=-\frac{A}{2} \alpha(\rho)
$$

with arbitrary constants $A, b, c$. The second-order symmetries have the form

$$
\begin{aligned}
& f_{2}=(A s+\bar{A}) \frac{s_{x x}}{s_{x}^{2}}+\frac{\alpha^{\prime}(\rho)}{4 \alpha^{2}}\left[(A s+\bar{A})\left(\frac{r_{x}}{s_{x}}-2\right)+(A r+\bar{C}) \frac{s_{x}}{r_{x}}\right]+b+a(s, r) s_{x}, \\
& g_{2}=(A r+\bar{C}) \frac{r_{x x}}{r_{x}^{2}}-\frac{\alpha^{\prime}(\rho)}{4 \alpha^{2}}\left[(A r+\bar{C})\left(\frac{s_{x}}{r_{x}}-2\right)+(A s+\bar{A}) \frac{r_{x}}{s_{x}}\right]+b+c(s, r) r_{x},
\end{aligned}
$$

with arbitrary constants $A, \bar{A}, \bar{C}, b$ and $a(s, r), c(s, r)$ satisfying (3.6).

In particular, if $c=0,(3.9)$ is satisfied for the physically interesting state equation of a polytropic gas with the parameters $a$ and $\gamma$ :

$$
P(\rho)=a^{2} \rho^{\gamma}, \quad \alpha(\rho)=a \sqrt{\gamma} \rho^{(\gamma-3) / 2} .
$$

(2) The function $\alpha(\rho)$ satisfies the condition $\alpha^{\prime}(\rho)=0$. This is the polytropic gas with $\gamma=3$. Then the second-order symmetries depend on arbitrary smooth functions $\psi_{1}, \psi_{2}$ :

$$
f_{2}=s_{x} \psi_{1}\left(\frac{s_{x x}}{s_{x}^{3}}, s\right), \quad g_{2}=r_{x} \psi_{2}\left(\frac{r_{x x}}{r_{x}^{3}}, r\right)
$$

The gas dynamics equations (3.2) have the form $s_{t}=-s s_{x}, r_{t}=-r r_{x}$. Their general solution $x-s t=F(s), x-r t=G(r)$ depends on two arbitrary smooth functions $F$ and $G$. Hence $s_{T}=r_{T}=0$ and the solution manifold consists solely of invariant solutions. 
(3) The function $\alpha(\rho)$ satisfies the condition $\alpha^{\prime}(\rho)=-(2 / \rho) \alpha(\rho)$. This is the Chaplygin gas [25] with the state equation

$$
P(\rho)=P_{0}-\frac{a^{2}}{\rho}, \quad \alpha(\rho)=\frac{a}{\rho^{2}} \quad\left(P_{0}>0\right)
$$

with the constants $P_{0}, a$. Then the set of second-order symmetries again depends on two arbitrary functions $\psi_{1}\left(s, s_{q}\right)$ and $\psi_{2}\left(r, r_{q}\right)$, where $q$ is the Lagrangian coordinate defined by the equation $d q=\rho d x-\rho u d t$ [27]. The diagonal form (3.2) of gas dynamics equations after transforming to the Lagrangian coordinates $q$, $t$ becomes $s_{t}=a s_{q}, r_{t}=$ $-a r_{q}$. Its general solution $s=F(q+a t), r=G(q-a t)$ depends on two arbitrary smooth functions $F$ and $G$. Since $x_{\tau}=t_{\tau}=0$ and hence $q_{\tau}=0$, we have $s_{\tau}=r_{\tau}=0$ as well and the solution manifold again consists solely of invariant solutions.

In the last two cases, the reason for the gas dynamics equations to be integrable in an explicit form is that the extent of arbitrariness of the set of invariant solutions and of the general solution set turns out to be the same: two arbitrary functions of one variable. Hence all (nonsingular) solutions are invariant.

\subsection{Recursion operators for gas dynamics equations}

THEOREM 3.2. First-order recursion operator for symmetries of the gas dynamics equations (3.2) is given by the formula

$$
R=\left(I D_{x}-\frac{\alpha_{x}(\rho)}{2 \alpha}\left(\begin{array}{cc}
1 & -1 \\
-1 & 1
\end{array}\right)\right)\left(\begin{array}{cc}
\frac{1}{s_{x}} & 0 \\
0 & \frac{1}{r_{x}}
\end{array}\right)
$$

It commutes with the operator of (3.3) on the solution set of (3.3)

$$
\left[I D_{t}+A, R\right]=0
$$

where the operator $D_{t}$ is calculated with the use of the gas dynamics equations.

COROLLARY 3.3. Operator (3.14) raises the order of higher symmetries by one unit:

$$
R\left(f_{N}, g_{N}\right)^{T}=\left(f_{N+1}, g_{N+1}\right)^{T} \quad(N=2,3, \ldots)
$$

and generates an infinite discrete set of higher symmetries of any order, for example,

$$
\left(\begin{array}{l}
f_{2} \\
g_{2}
\end{array}\right)=R\left(\begin{array}{l}
1 \\
1
\end{array}\right)=R^{2} x\left(\begin{array}{l}
s \\
r
\end{array}\right)_{x}
$$

COROLLARY 3.4. The solution of the recursion relation (3.16) has the form

$$
\left(\begin{array}{l}
f_{N} \\
g_{N}
\end{array}\right)=R^{N-1}\left(\begin{array}{l}
1 \\
1
\end{array}\right)=R^{N} x\left(\begin{array}{l}
s \\
r
\end{array}\right)_{x}, \quad N=2,3, \ldots
$$


Formula (3.14) gives us a general form of the first-order recursion operator for a generic function $\alpha(\rho)$. If the function $\alpha(\rho)$ satisfies the equation

$$
\left(\frac{\alpha^{\prime}}{\alpha^{2}}\right)^{\prime}[A(r-s)+\bar{c}-\bar{a}]=-2 A \frac{\alpha^{\prime}}{\alpha}
$$

with arbitrary constants $A, \bar{a}, \bar{c}$, then a more general form of the recursion operator is obtained. In particular, (3.19) is satisfied by the state equation (3.11) of the polytropic gas if the constants satisfy the equations

$$
A=\frac{-(\gamma-1)(\bar{a}-\bar{c})}{4 a \sqrt{\gamma} \rho_{0}^{(\gamma-1) / 2}} \quad\left(\rho_{0} \neq 0\right) \quad \text { or } \quad \bar{a}=\bar{c} \quad\left(\rho_{0}=0\right)
$$

THEOREM 3.5. Equations of the polytropic gas admit first-order recursion operator depending upon one essential arbitrary constant $\bar{a} / \bar{c}\left(\rho_{0} \neq 0\right)$ or $\bar{a} / A\left(\rho_{0}=0\right)$ of the form

$$
\begin{aligned}
R=\{ & \left(\begin{array}{cc}
A s+\bar{a} & 0 \\
0 & A r+\bar{c}
\end{array}\right) D_{x}-\frac{\alpha^{\prime}(\rho)}{4 \alpha^{2}} \\
& \left.\cdot\left(\begin{array}{cc}
(A s+\bar{a})\left(r_{x}-s_{x}\right) & -\left[(A s+\bar{a}) r_{x}-(A r+\bar{c}) s_{x}\right] \\
-\left[(A s+\bar{a}) r_{x}-(A r+\bar{c}) s_{x}\right] & (A r+\bar{c})\left(r_{x}-s_{x}\right)
\end{array}\right)\right\} \\
& \times\left(\begin{array}{cc}
\frac{1}{s_{x}} & 0 \\
0 & \frac{1}{r_{x}}
\end{array}\right),
\end{aligned}
$$

where $\alpha^{\prime}(\rho) /\left(4 \alpha^{2}\right)=(\gamma-3) /\left[8 a \sqrt{\gamma} \rho^{(\gamma-1) / 2}\right]$ and $A, \bar{a}, \bar{c}$ satisfy (3.20).

Now consider the action of the recursion operator (3.14) on the subspace of hydrodynamic symmetries (3.5) subject to conditions (3.6):

$$
R\left(\begin{array}{l}
a(s, r) s_{x} \\
c(s, r) r_{x}
\end{array}\right)=\left(\begin{array}{l}
a_{1}(s, r) s_{x} \\
c_{1}(s, r) r_{x}
\end{array}\right)
$$

Here the functions $a_{1}, c_{1}$ are generated from $a, c$ by the transformation

$$
a_{1}(s, r)=a_{s}(s, r)+\left[\frac{\alpha^{\prime}(\rho)}{\left(4 \alpha^{2}\right)}\right](a-c), \quad c_{1}(s, r)=c_{r}(s, r)+\left[\frac{\alpha^{\prime}(\rho)}{\left(4 \alpha^{2}\right)}\right](a-c) .
$$

COROLlaRY 3.6. Transformation (3.23) is a recursion for solutions of the linear system (3.6), that is, if $a(s, r), c(s, r)$ is its solution, then $a_{1}(s, r), c_{1}(s, r)$ is also the solution.

From (3.22),

$$
R^{N}\left(\begin{array}{l}
a(s, r) s_{x} \\
c(s, r) r_{x}
\end{array}\right)=\left(\begin{array}{l}
a_{N}(s, r) s_{x} \\
c_{N}(s, r) r_{x}
\end{array}\right) \quad(N=1,2, \ldots)
$$

where the solution $a_{N}(s, r), c_{N}(s, r)$ of (3.6) is a result of the $N$-fold application of transformation (3.23) to the solution $a(s, r), c(s, r)$. 
3.3. Generalized gas dynamics equations, their symmetries, and recursion operators. Lie equations for hydrodynamic symmetries (3.5) of gas dynamics equations (3.2) are

$$
s_{\tau}=a(s, r) s_{x}, \quad r_{\tau}=c(s, r) r_{x}
$$

with $a, c$ subject to conditions (3.6). Change the notation $a(s, r), c(s, r)$ to $\phi(s, r)$, $\psi(s, r)$ and the group parameter $\tau$ to the new time variable $t$. We obtain the systems of the form (2.1) subject to the additional constraints

$$
s_{t}=\phi(s, r) s_{x}, \quad r_{t}=\psi(s, r) r_{x}, \quad \phi_{r}(s, r)=\psi_{s}(s, r)=\left[\frac{\alpha^{\prime}(\rho)}{4 \alpha^{2}}\right](\phi-\psi),
$$

that is, $(\phi, \psi)$ is an arbitrary smooth solution of the linear system (3.6).

Equations (3.26) appeared for the first time in [30,31] and later Olver and Nutku had called them generalized gas dynamics (GGD) equations [24]. They also pointed out many interesting applications of these equations in physics.

The determining equation for symmetries of any of GGD systems (3.26) has the form

$$
\left(I D_{t}+A_{(\phi, \psi)}\right)(f, g)^{T}=(0,0)^{T},
$$

where the operator $A_{(\phi, \psi)}$ is defined by the formula

$$
A_{(\phi, \psi)}=-\left(\begin{array}{cc}
\phi & 0 \\
0 & \psi
\end{array}\right) D_{x}-\left(\begin{array}{cc}
s_{x} & 0 \\
0 & r_{x}
\end{array}\right)\left\{\left(\begin{array}{cc}
\phi_{1} & 0 \\
0 & \psi_{1}
\end{array}\right)-\frac{\alpha^{\prime}(\rho)}{4 \alpha^{2}}(\phi-\psi)\left(\begin{array}{cc}
1 & -1 \\
-1 & 1
\end{array}\right)\right\}
$$

with $\phi_{1}, \psi_{1}$ generated from $\phi, \psi$ by (3.23) and hence satisfying (3.26):

$$
\begin{aligned}
& \phi_{1}(s, r)=\phi_{s}(s, r)+\left[\frac{\alpha^{\prime}(\rho)}{\left(4 \alpha^{2}\right)}\right](\phi-\psi), \\
& \psi_{1}(s, r)=\psi_{r}(s, r)+\left[\frac{\alpha^{\prime}(\rho)}{\left(4 \alpha^{2}\right)}\right](\phi-\psi) .
\end{aligned}
$$

THEOREM 3.7. For the GGD equations (3.26), all the hydrodynamic symmetries homogeneous in derivatives with no explicit dependence on $x, t$ are generated by the Lie equations (3.25) with the coefficients $a(s, r)$ and $c(s, r)$ satisfying the linear system (3.6) and hence coincide with the hydrodynamic symmetries of the gas dynamics equations.

Thus, the hydrodynamic symmetries of any GGD system are generated by the Lie equations which belong to the same GGD hierarchy but have a different time variable $\tau$.

THEOREM 3.8. Operator $R$ defined by formula (3.14) is a recursion operator for symmetries of the whole infinite GGD hierarchy (3.26). It commutes with the operator of the determining equation (3.27) on the solution manifold of the latter equation

$$
\left[I D_{t}+A_{(\phi, \psi)}, R\right]=0
$$

where the operator $D_{t}$ is calculated with the use of GGD equations. 
THEOREM 3.9. All the hydrodynamic symmetries of the generalized gas dynamics equations (3.26) with the coefficients $\phi(s, r), \psi(s, r)$ are generated by the Lie equations

$$
\begin{aligned}
& s_{T}=a s_{x}-\lambda(x+t \phi) s_{x}+c_{0}\left(1+t \phi_{1} s_{x}\right), \\
& r_{T}=c r_{x}-\lambda(x+t \psi) r_{x}+c_{0}\left(1+t \psi_{1} r_{x}\right),
\end{aligned}
$$

where $a=a(s, r), c=c(s, r)$ satisfy (3.6) and $\lambda, c_{0}$ are arbitrary constants.

THEOREM 3.10. Generalized gas dynamics equations with the coefficients $\phi(s, r)$, $\psi(s, r)$ have an infinite discrete set of higher symmetries of any order $N=2,3, \ldots$ with a functional arbitrariness and an explicit dependence on $t$.

Symmetries of the order $N=2$ are generated by the Lie equations

$$
\begin{aligned}
& s_{T}=a_{1} s_{x}-\lambda\left(1+t \phi_{1} s_{x}\right)+c_{0}\left(t \phi_{2} s_{x}+f_{2}\right), \\
& r_{T}=c_{1} r_{x}-\lambda\left(1+t \psi_{1} r_{x}\right)+c_{0}\left(t \psi_{2} r_{x}+g_{2}\right) .
\end{aligned}
$$

Here the functions $a_{1}(s, r), c_{1}(s, r)$ and $\phi_{1}(s, r), \psi_{1}(s, r)$ are obtained by the transformations (3.23) and (3.29) from $a(s, r), c(s, r)$ and $\phi(s, r), \psi(s, r)$, respectively. The functions $\phi_{2}(s, r), \psi_{2}(s, r)$ are obtained by a two-fold application of the transformation (3.29) to $\phi, \psi$, and the functions $a_{1}(s, r), c_{1}(s, r)$ form an arbitrary smooth solution of (3.6). The functions $f_{2}, g_{2}$ are defined by (3.7). Symmetries of order $N+1(N+1 \geq 3)$ are generated by the Lie equations

$$
\left(\begin{array}{l}
s \\
r
\end{array}\right)_{\tau}=\left(\begin{array}{c}
a_{N} s_{x} \\
c_{N} r_{x}
\end{array}\right)-\lambda\left\{\left(\begin{array}{l}
f_{N} \\
g_{N}
\end{array}\right)+t\left(\begin{array}{l}
\phi_{N} s_{x} \\
\psi_{N} r_{x}
\end{array}\right)\right\}+c_{0}\left\{\left(\begin{array}{l}
f_{N+1} \\
g_{N+1}
\end{array}\right)+t\left(\begin{array}{c}
\phi_{N+1} s_{x} \\
\psi_{N+1} r_{x}
\end{array}\right)\right\} .
$$

Here $a_{N}=a_{N}(s, r), c_{N}=c_{N}(s, r), \phi_{N}=\phi_{N}(s, r), \psi_{N}=\psi_{N}(s, r)$, the subscript $N$ denotes $N$-fold application of the transformations (3.23) and (3.29), the functions $f_{N}, g_{N}$ are defined by formula (3.18) with their explicit form for $N=2,3$ given by formulas (3.7) and (3.8), and $\lambda, c_{0}$ are arbitrary constants.

3.4. Noncommutative algebra of higher symmetries of gas dynamics equations. Denote by $\hat{X}_{(a, c)}$ the evolutionary representatives [23] of generators of the hydrodynamic symmetries corresponding to the Lie equations (3.25) subject to condition (3.6). Let $\hat{X}_{N}$ denote the evolutionary representatives of generators of $N$ th-order higher symmetries (3.18) of the gas dynamics equations for $N=2,3, \ldots$.

THEOREM 3.11. Hydrodynamic and higher symmetries of the gas dynamics equations generate an infinite-dimensional noncommutative Lie algebra in which the hydrodynamic symmetries form an infinite-dimensional commutative ideal

$$
\left[\hat{X}_{(a, c)}, \hat{X}_{(\tilde{a}, \tilde{c})}\right]=0, \quad\left[\hat{X}_{N}, \hat{X}_{(a, c)}\right]=\hat{X}_{\left(a_{N}, c_{N}\right)}, \quad\left[\hat{X}_{M}, \hat{X}_{N}\right]=0,
$$

where $M, N=2,3, \ldots, a, c$ and $\tilde{a}, \tilde{c}$ satisfy (3.6), and $a_{N}, c_{N}$ are defined by (3.24).

THEOREM 3.12. Let $N=2,3, \ldots$. The generalized gas dynamics equations have the common infinite set of higher symmetries (3.33) of the order greater than or equal to $N+1$ if and only if the right-hand sides of these equations differ only by a term belonging 
to the kernel of the operator $R^{N}$, that is, $\phi_{N}, \psi_{N}$ coincide for all these equations. If the right-hand sides $\phi s_{x}, \psi r_{x}$ of GGD equations (3.26) themselves belong to the kernel of the operator $R^{N}$, then for all such equations, the generators $\hat{X}_{(\phi, \psi)}$ commute with all the generators $\hat{X}_{n}$ of the higher symmetries (3.18) for $n=N, N+1, \ldots$.

COROLLARY 3.13. Commutative symmetry subalgebras for the gas dynamics equations are formed by the generators $\hat{X}_{(a, c)}$ of such hydrodynamic symmetries whose characteristics $\left(a s_{x}, c r_{x}\right)$ belong to a kernel of some integer power $R^{N}$ of the recursion operator for $N \geq 2$ and by the generators $\hat{X}_{n}$ of higher symmetries (3.18) of the order $n \geq N$.

Thus, according to Corollary 3.13, a problem of a constructive description of the kernel of $R^{N}$ naturally arises. It is solved by means of the inverse recursion operator $R^{-1}$ :

$$
R^{-1}=\left(\begin{array}{cc}
s_{x} & 0 \\
0 & r_{x}
\end{array}\right)\left\{I+\frac{\alpha}{2}\left(\begin{array}{cc}
1 & -1 \\
-1 & 1
\end{array}\right) D_{x}^{-1} \frac{\alpha_{x}}{\alpha^{2}}\right\} D_{x}^{-1}
$$

where $D_{x}^{-1}=\int d x$ is the operator of integration with respect to $x$ at a constant value of $t$ with the integration "constant" $c(t)$ depending on $t$. Hence a kernel of $R$ has the form

$$
R^{-1}\left(\begin{array}{l}
0 \\
0
\end{array}\right)=c_{1}(t)\left(\begin{array}{l}
s_{x} \\
r_{x}
\end{array}\right)+c_{2}(t) \alpha(\rho)\left(\begin{array}{c}
-s_{x} \\
r_{x}
\end{array}\right) .
$$

A kernel of $R^{2}$ is given by the formula

$$
\begin{aligned}
& R^{-2}\left(\begin{array}{l}
0 \\
0
\end{array}\right)=c_{1}(t)\left(\begin{array}{c}
(u-\rho \alpha(\rho)) s_{x} \\
(u+\rho \alpha(\rho)) r_{x}
\end{array}\right)+c_{2}(t)\left(\begin{array}{l}
{\left[\int \alpha^{2}(\rho) d \rho-u \alpha(\rho)\right] s_{x}} \\
{\left[\int \alpha^{2}(\rho) d \rho+u \alpha(\rho)\right] r_{x}}
\end{array}\right) \\
& +c_{3}(t)\left(\begin{array}{c}
s_{x} \\
r_{x}
\end{array}\right)+c_{4}(t) \alpha(\rho)\left(\begin{array}{c}
-s_{x} \\
r_{x}
\end{array}\right) \text {, }
\end{aligned}
$$

where $c_{i}(t)$ are arbitrary smooth functions.

3.5. Lax representation and invariant solutions of generalized gas dynamics equations. Formula (3.15) implies the Lax representation [18] of the gas dynamics equations

$$
\frac{\partial R}{\partial t}=[R, A]
$$

where the recursion operator (3.14) and the "stationary part" (3.4) of the operator of the determining equation for symmetries (3.3) form the Lax pair of the Ibragimov-Shabat type [16, 17, 21]. Equation (3.30) gives the Lax representation for any generalized gas dynamics equations (3.26) with the change of $A$ to $A_{(\phi, \psi)}$ defined by (3.28):

$$
\frac{\partial R}{\partial t}=\left[R, A_{(\phi, \psi)}\right] .
$$


In 1982, Faddeev and Kulish in a private communication pointed out to the author that these Lax pairs were degenerate, that is, the mapping of a potential to the scattering data was singular so that the method of the inverse scattering transform could not be applied. However, it is not a bad feature of GGD equations since they can be linearized in a classical sense by the hodograph transformation. The Lax representation for these equations is still useful for the construction of their invariant solutions by means of the inverse recursion operator (3.35) [30].

Consider solutions of the gas dynamics equations (3.1) invariant with respect to the higher symmetries given by (3.18):

$$
\left(f_{N}, g_{N}\right)^{T}=R^{N-1}(1,1)^{T}=0, \quad N=2,3, \ldots
$$

Define the matrix $U$ and the matrix-integral operator $K$ by the formulas

$$
U=\left(\begin{array}{cc}
u & \int_{0}^{\rho} \alpha^{2}(\rho) d \rho \\
\rho & u
\end{array}\right), \quad K=D_{x}^{-1} U_{x}=D_{x}^{-1}\left(\begin{array}{cc}
u_{x} & \alpha^{2}(\rho) \rho_{x} \\
\rho_{x} & u_{x}
\end{array}\right)
$$

where we define $D_{x}^{-1}=\int d x$ so that all the integration constants $c_{i}$ do not depend on $t$.

THEOREM 3.14. For any $N=2,3, \ldots$, the equations

$$
\begin{aligned}
K^{N-1}\left(\begin{array}{l}
c_{1} \\
c_{2}
\end{array}\right) \equiv & \left(\begin{array}{c}
c_{2 N-1} \\
c_{2 N}
\end{array}\right)+c_{2 N-2}\left(\begin{array}{c}
\alpha^{2}(\rho) d \rho \\
u
\end{array}\right)+c_{2 N-3}\left(\begin{array}{c}
u \\
\rho
\end{array}\right) \\
& +c_{2 N-4}\left(\begin{array}{c}
u \int \alpha^{2}(\rho) d \rho \\
\frac{u^{2}}{2}+\int d \rho \int \alpha^{2}(\rho) d \rho
\end{array}\right)+c_{2 N-5}\left(\begin{array}{c}
\left.\frac{u^{2}}{2}+\int \alpha^{2}(\rho) \rho d \rho\right)+\cdots \\
u \rho
\end{array}\right)+ \\
= & \left(\begin{array}{c}
x-u t \\
-\rho t
\end{array}\right)
\end{aligned}
$$

determine in the implicit form $t=t(u, \rho), x=x(u, \rho)$ an infinite set of exact solutions $u=u(x, t), \rho=\rho(x, t)$ of the gas dynamics equations (3.1) which are invariant with respect to the higher symmetries (3.18).

For $N=1$, formula (3.42) becomes $c_{1}=x-u t, c_{2}=-\rho t$ and gives a trivial solution for which $u_{t}+u u_{x}=0, \rho_{t}+\rho u_{x}=0$, that is, the velocity and density $u$ and $\rho$ are constant in the Lagrangian frame which moves together with a gas particle. For $N=2$, formula (3.42) takes the form $u-u_{0}=\bar{\rho}\left(t-t_{0}\right), \bar{\rho}\left(t-t_{0}\right)^{2}-\int_{\bar{\rho}_{0}}^{\bar{\rho}} P^{\prime}(\lambda \bar{\rho})\left(1 / \bar{\rho}^{2}\right) d \bar{\rho}=x-x_{0}-u_{0}\left(t-t_{0}\right)$, where $\bar{\rho}=\rho / \lambda$ and $\lambda, u_{0}, x_{0}, t_{0}$, and $\bar{\rho}_{0}$ are constants. It describes the motion of a piston in a gas flow after the explosion.

Now consider invariant solutions of the generalized gas dynamics equations expressed through the variables $u, \rho$ :

$$
u_{t}=w(u, \rho) u_{x}+v(u, \rho) \alpha^{2}(\rho) \rho_{x}, \quad \rho_{t}=v(u, \rho) u_{x}+w(u, \rho) \rho_{x},
$$


where the coefficients $v, w$ satisfy the linear system $w_{u}=v_{\rho}, w_{\rho}=\alpha^{2}(\rho) v_{u}$. Corollary 3.13 implies that the higher symmetries (3.18) of gas dynamics equations of the orders $N_{0}, N_{0}+1, \ldots$ are also higher symmetries of those GGD equations (3.26) whose righthand sides belong to a kernel of the recursion operator $R^{N_{0}}$, that is, $\phi_{N_{0}}=\psi_{N_{0}}=0$. We give now their explicit form allowing them to depend explicitly on $t$ :

$$
\begin{aligned}
& D_{t}\left(\begin{array}{l}
u \\
\rho
\end{array}\right)=\left(\begin{array}{cc}
u_{x} & \alpha^{2}(\rho) \rho_{x} \\
\rho_{x} & u_{x}
\end{array}\right) K^{N_{0}-1}\left(\begin{array}{l}
a_{1}(t) \\
a_{2}(t)
\end{array}\right) \equiv\left(\begin{array}{cc}
u_{x} & \alpha^{2}(\rho) \rho_{x} \\
\rho_{x} & u_{x}
\end{array}\right) \\
& \cdot\left\{\left(\begin{array}{c}
a_{2 N_{0}-1}(t) \\
a_{2 N_{0}}(t)
\end{array}\right)+a_{2 N_{0}-2}(t)\left(\int \begin{array}{c}
\alpha^{2}(\rho) d \rho \\
u
\end{array}\right)+a_{2 N_{0}-3}(t)\left(\begin{array}{l}
u \\
\rho
\end{array}\right)\right. \\
& +a_{2 N_{0}-4}(t)\left(\begin{array}{c}
u \int \alpha^{2}(\rho) d \rho \\
\frac{u^{2}}{2}+\int d \rho \int \alpha^{2}(\rho) d \rho
\end{array}\right) \\
& \left.+a_{2 N_{0}-5}(t)\left(\begin{array}{c}
\frac{u^{2}}{2}+\int \alpha^{2}(\rho) \rho d \rho \\
u \rho
\end{array}\right)+\cdots\right\} \text {. }
\end{aligned}
$$

Here in definition (3.41) of operator $K$, the "constants" $a_{i}(t)$ of the integration with respect to $x$ may depend on $t$ as arbitrary smooth functions. Consider solutions of GGD equations (3.44) invariant with respect to the higher symmetries (3.18).

THEOREM 3.15. For any $N=N_{0}, N_{0}+1, \ldots$ with $N_{0} \geq 2$, the equations

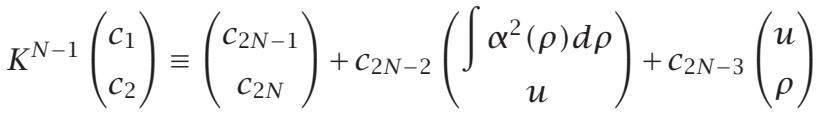

$$
\begin{aligned}
& +c_{2 N-4}\left(\begin{array}{c}
u \int \alpha^{2}(\rho) d \rho \\
\frac{u^{2}}{2}+\int d \rho \int \alpha^{2}(\rho) d \rho
\end{array}\right)+c_{2 N-5}\left(\begin{array}{c}
\frac{u^{2}}{2}+\int \alpha^{2}(\rho) \rho d \rho \\
u \rho
\end{array}\right)+\cdots \\
& =\left(\begin{array}{l}
x \\
0
\end{array}\right)+\int_{0}^{t}\left(\begin{array}{c}
a_{2 N_{0}-1}(t) \\
a_{2 N_{0}}(t)
\end{array}\right) d t+\left(\int \begin{array}{c}
\alpha^{2}(\rho) d \rho \\
u
\end{array}\right) \int_{0}^{t} a_{2 N_{0}-2}(t) d t \\
& +\left(\begin{array}{l}
u \\
\rho
\end{array}\right) \int_{0}^{t} a_{2 N_{0}-3}(t) d t+\left(\begin{array}{c}
u \int \alpha^{2}(\rho) d \rho \\
\frac{u^{2}}{2}+\int d \rho \int \alpha^{2}(\rho) d \rho
\end{array}\right) \int_{0}^{t} a_{2 N_{0}-4}(t) d t \\
& +\left(\begin{array}{c}
\frac{u^{2}}{2}+\int \alpha^{2}(\rho) \rho d \rho \\
u \rho
\end{array}\right) \int_{0}^{t} a_{2 N_{0}-5}(t) d t+\cdots
\end{aligned}
$$

determine an infinite set of exact solutions $u=u(x, t), \rho=\rho(x, t)$ of the generalized gas dynamics equations (3.44) which may explicitly depend on $t$. These solutions are invariant with respect to the Nth-order higher symmetries (3.18) of these equations. 
Here the functions $a_{i}(t)$ and the constants $c_{i}$ with $i \leq 0$ must be put equal to zero. For the gas dynamics equations (3.1), $a_{2 N_{0}-3}(t)=-1, a_{i}(t)=0$ for $i \neq 2 N_{0}-3$.

\section{Separable two-component Hamiltonian systems}

4.1. Hamiltonian structure of generalized gas dynamics equations. Consider twocomponent Hamiltonian systems of the hydrodynamic type of the form

$$
D_{t}\left(\begin{array}{c}
u \\
\rho
\end{array}\right)=\sigma_{1} D_{x}\left(\begin{array}{l}
H_{u}(u, \rho) \\
H_{\rho}(u, \rho)
\end{array}\right), \quad \sigma_{1}=\left(\begin{array}{ll}
0 & 1 \\
1 & 0
\end{array}\right)
$$

Here $H(u, \rho)$ is the Hamiltonian density of the hydrodynamic type which corresponds to the Hamiltonian $\mathscr{H}=\int_{-\infty}^{\infty} H(u, \rho) d x$. For shortness, we will also call $H(u, \rho)$ a Hamiltonian. Equations (4.1) take the form of the Hamilton equations

$$
D_{t}\left(\begin{array}{c}
u \\
\rho
\end{array}\right)=\left\{\left(\begin{array}{c}
u \\
\rho
\end{array}\right), H\right\}
$$

with the Poisson bracket of the hydrodynamic type [4]

$$
\{H, h\}=\left(h_{u}, h_{\rho}\right) D_{x} \sigma_{1}\left(H_{u}, H_{\rho}\right)^{T} .
$$

Define the Hamiltonian matrix

$$
\hat{H}=\left(\begin{array}{ll}
H_{\rho u} & H_{\rho \rho} \\
H_{u u} & H_{u \rho}
\end{array}\right)
$$

so that (4.1) takes the form

$$
\left(\begin{array}{c}
u \\
\rho
\end{array}\right)_{t}=\hat{H}\left(\begin{array}{l}
u \\
\rho
\end{array}\right)_{x} \Longleftrightarrow\left(I D_{t}-\hat{H} D_{x}\right)\left(\begin{array}{l}
u \\
\rho
\end{array}\right)=\left(\begin{array}{l}
0 \\
0
\end{array}\right)
$$

The gas dynamics equations (3.1) have the Hamiltonian form (4.1) and (4.5) with the Hamiltonian

$$
H(u, \rho)=-\left[\frac{\rho u^{2}}{2}+\int_{0}^{\rho} d \rho \int_{0}^{\rho} \alpha^{2}(\rho) \rho d \rho\right]
$$

The same is valid for the generalized gas dynamics equations (3.44) with the Hamiltonians $H(u, \rho, t)$ that may depend explicitly on $t$ defined by the formula

$$
\left(\begin{array}{l}
H_{\rho} \\
H_{u}
\end{array}\right)=K^{N_{0}}\left(\begin{array}{l}
a_{1}(t) \\
a_{2}(t)
\end{array}\right)
$$


with the operator $K$ defined by (3.41). For example, if $N_{0}=2$, (4.7) becomes

$$
\begin{aligned}
H(u, \rho, t)= & a_{1}(t)\left[\frac{\rho u^{2}}{2}+\int_{0}^{\rho} d \rho \int_{0}^{\rho} \alpha^{2}(\rho) \rho d \rho\right]+a_{2}(t)\left[\frac{u^{3}}{3 !}+u \int_{0}^{\rho} d \rho \int_{0}^{\rho} \alpha^{2}(\rho) d \rho\right] \\
& +a_{3}(t) u \rho+a_{4}(t)\left[\frac{u^{2}}{2}+\int_{0}^{\rho} d \rho \int_{0}^{\rho} \alpha^{2}(\rho) d \rho\right]+a_{5}(t) \rho+a_{6}(t) u .
\end{aligned}
$$

\subsection{Separable Hamiltonian systems}

DEFINITION 4.1. It is said that the Hamiltonians $\int_{-\infty}^{\infty} H d x$ and $\int_{-\infty}^{\infty} h d x$ commute if the Poisson bracket (4.3) is the exact total derivative with respect to $x$ :

$$
\{H(u, \rho, t), h(u, \rho, t)\}=D_{x}[Q(u, \rho, t)]
$$

Then the Hamiltonian matrices $\hat{H}$ and $\hat{h}$ defined by (4.4) commute: $[\hat{H}, \hat{h}]=0$, and also it is said that the Hamiltonians $H$ and $h$ commute.

THEOREM 4.2. For any hydrodynamic-type Hamiltonian $H(u, \rho)$, there exists an infinite set of Hamiltonians $h(u, \rho)$ which commute with it and with each other, and are arbitrary solutions of the wave equation $H_{\rho \rho} h_{u u}-h_{\rho \rho} H_{u u}=0$, or with the notation $V(u, \rho)=H_{u u} / H_{\rho \rho}$ for $H_{u u} H_{\rho \rho} \neq 0$,

$$
h_{u u}-V(u, \rho) h_{\rho \rho}=0 .
$$

DEFINITION 4.3. Systems (4.5) for which the wave equation (4.10) admits a separation of variables $u, \rho$, that is, $V(u, \rho)=\beta^{2}(u) / \alpha^{2}(\rho)$, are called separable Hamiltonian systems [24, 31]. Their Hamiltonians $H$ and all the Hamiltonians $h$ commuting with $H$ satisfy the same equation

$$
\left(\frac{1}{\beta^{2}(u)}\right) H_{u u}=\left(\frac{1}{\alpha^{2}(\rho)}\right) H_{\rho \rho}, \quad\left(\frac{1}{\beta^{2}(u)}\right) h_{u u}=\left(\frac{1}{\alpha^{2}(\rho)}\right) h_{\rho \rho} .
$$

Gas dynamics equations (3.1) and GGD equations (3.26) with the Hamiltonians (4.6) and (4.7) are examples of separable systems with $\beta^{2}(u)=1$.

DEFINITION 4.4. If $H(u, \rho)$ is a Hamiltonian and $H_{u u} / H_{\rho \rho}=1 / \alpha^{2}(\rho)$ is a function of $\rho$ only $(\beta(u)=1)$, then $H$ is called the Hamiltonian of the generalized gas dynamics.

Physical applications of the separable Hamiltonian systems are given in [24].

4.3. Second-order recursion operator and Lax representation for separable Hamiltonian systems. We show that separable Hamiltonian systems obtained by the Manin's construction possess higher symmetries, a recursion operator, the Lax representation of the Ibragimov-Shabat type, and good integrability properties [20, 31].

We introduce the notation $\partial_{u}=\partial / \partial u, \partial_{\rho}=\partial / \partial \rho, \partial_{u}^{-1}=\int_{0}^{u} d u$, and $\partial_{\rho}^{-1}=\int_{0}^{\rho} d \rho$, where the integration with respect to one variable is performed at a constant value of another variable. The Manin's construction gives rise to the two fundamental series of mutually 
commuting Hamiltonians [31]

$$
\begin{aligned}
H^{(2 m)}(1,0) & =\sum_{n=0}^{m}\left(\partial_{u}^{-2} \beta^{2}\right)^{m-n}\left(\partial_{\rho}^{-2} \alpha^{2}\right)^{n} \rho, \\
H^{(2 m-1)}(1,0) & =\sum_{n=0}^{m}\left(\partial_{u}^{-2} \beta^{2}\right)^{m-n-1}\left(\partial_{\rho}^{-2} \alpha^{2}\right)^{n} u \rho, \\
H^{(2 m)}(0,1) & =\sum_{n=0}^{m}\left(\partial_{u}^{-2} \beta^{2}\right)^{m-n}\left(\partial_{\rho}^{-2} \alpha^{2}\right)^{n} u, \\
H^{(2 m-1)}(0,1) & =\sum_{n=0}^{m}\left(\partial_{u}^{-2} \beta^{2}\right)^{m-n}\left(\partial_{\rho}^{-2} \alpha^{2}\right)^{n},
\end{aligned}
$$

where $\beta=\beta(u), \alpha=\alpha(\rho), m=0,1,2, \ldots, H^{(-1)}(1,0)=0$, and $H^{(-1)}(0,1)=1$. Here every operator of $\partial_{u}^{-1}, \partial_{\rho}^{-1}$ acts on all the factors standing to the right of it; for example,

$$
\left(\partial_{u}^{-2} \beta^{2}(u)\right)^{2}=\int_{0}^{u} d u \int_{0}^{u}\left[\beta^{2}(u) \int_{0}^{u} d u \int_{0}^{u} \beta^{2}(u) d u\right] d u
$$

For arbitrary constants $c_{1}, c_{2}$, we define

$$
H^{(N)}\left(c_{1}, c_{2}\right)=c_{1} H^{(N)}(1,0)+c_{2} H^{(N)}(0,1) .
$$

The basic Hamiltonians for the GGD equations (3.44) are obtained at $\beta(u)=1$ :

$$
\begin{aligned}
& H^{(N)}(1,0)=\sum_{n=0}^{[N / 2]} \frac{u^{N-2 n}}{(N-2 n) !}\left(\partial_{\rho}^{-2} \alpha^{2}(\rho)\right)^{n} \rho \\
& H^{(N)}(0,1)=\sum_{n=0}^{[(N+1) / 2]} \frac{u^{N+1-2 n}}{(N+1-2 n) !}\left(\partial_{\rho}^{-2} \alpha^{2}(\rho)\right)^{n} .
\end{aligned}
$$

We list explicitly several Hamiltonians from series (4.12):

$$
\begin{aligned}
& H^{(0)}(1,0)=\rho, \\
& H^{(0)}(0,1)=u, \\
& H^{(1)}(1,0)=u \rho, \\
& H^{(1)}(0,1)=\partial_{u}^{-2} \beta^{2}+\partial_{\rho}^{-2} \alpha^{2}, \\
& H^{(2)}(1,0)=\left(\partial_{u}^{-2} \beta^{2}(u)+\partial_{\rho}^{-2} \alpha^{2}(\rho)\right) \rho, \\
& H^{(3)}(1,0)=\left(\partial_{u}^{-2} \beta^{2}(u)+\partial_{\rho}^{-2} \alpha^{2}(\rho)\right) u \rho, \\
& H^{(2)}(0,1)=\left(\partial_{u}^{-2} \beta^{2}+\partial_{\rho}^{-2} \alpha^{2}\right) u, \\
& H^{(3)}(0,1)=\left(\partial_{u}^{-2} \beta^{2}\right)^{2}+\partial_{u}^{-2} \beta^{2} \partial_{\rho}^{-2} \alpha^{2}+\left(\partial_{\rho}^{-2} \alpha^{2}\right)^{2} .
\end{aligned}
$$


In particular, for the Hamiltonians (4.15) of the generalized gas dynamics [24]:

$$
\begin{gathered}
H^{(2)}(1,0)=\frac{\rho u^{2}}{2}+\tilde{G}_{1}(\rho), \\
H^{(3)}(1,0)=\frac{\rho u^{3}}{6}+u \tilde{G}_{1}(\rho), \\
H^{(1)}(0,1)=\frac{u^{2}}{2}+G_{1}(\rho), \quad H^{(2)}(0,1)=\frac{u^{3}}{6}+u G_{1}(\rho), \\
\tilde{G}_{1}(\rho) \stackrel{\text { def }}{=} \int_{0}^{\rho}(\rho-\sigma) \sigma \alpha^{2}(\sigma) d \sigma, \quad G_{1}(\rho) \stackrel{\text { def }}{=} \int_{0}^{\rho}(\rho-\sigma) \alpha^{2}(\sigma) d \sigma .
\end{gathered}
$$

Consider the special case $\beta(u)=1$ of the generalized gas dynamics Hamiltonians. Define the integral-matrix operator $K_{0}$ using the matrix $U$ defined by (3.41):

$$
\begin{aligned}
K_{0} & =\partial_{x}^{-1} U_{x}=\int_{0}^{x} d x\left(\begin{array}{cc}
u_{x} & \alpha^{2}(\rho) \rho_{x} \\
\rho_{x} & u_{x}
\end{array}\right) \\
& =\int_{(0,0)}^{(u, \rho)} U_{u} d u+U_{\rho} d \rho=\partial_{u}^{-1} I+\partial_{\rho}^{-1}\left(\begin{array}{cc}
0 & \alpha^{2}(\rho) \\
1 & 0
\end{array}\right) .
\end{aligned}
$$

Here the integral with respect to $x$ is taken at a constant value of $t$ and the latter integral operator is the curvilinear integral independent of the integration path in the $(u, \rho)$ plane.

THEOREM 4.5. Generalized gas dynamics Hamiltonians of series (4.15) and their combinations $H^{(N)}\left(c_{1}, c_{2}\right)$ defined by (4.14) satisfy the recursion relation

$$
\left(H_{\rho}^{(N+1)}, H_{u}^{(N+1)}\right)^{T}=K_{0}\left(H_{\rho}^{(N)}, H_{u}^{(N)}\right)^{T}, \quad N=0,1,2, \ldots,
$$

with the recursion operator $K_{0}$ defined by (4.18) and the solution

$$
\left(H_{\rho}^{(N)}, H_{u}^{(N)}\right)^{T}=K_{0}^{N}\left(H_{\rho}^{(0)}, H_{u}^{(0)}\right)^{T}=K_{0}^{N}\left(c_{1}, c_{2}\right)^{T}, \quad H^{(N)} \stackrel{\text { def }}{=} H^{(N)}\left(c_{1}, c_{2}\right) .
$$

In the general case with arbitrary functions $\beta(u)$ and $\alpha(\rho)$, we define the matrices

$$
U_{1}=\left(\begin{array}{cc}
u & \partial_{\rho}^{-1} \alpha^{2}(\rho) \\
\rho & \partial_{u}^{-1} \beta^{2}(u)
\end{array}\right), \quad U_{2}=\left(\begin{array}{cc}
\partial_{u}^{-1} \beta^{2}(u) & \partial_{\rho}^{-1} \alpha^{2}(\rho) \\
\rho & u
\end{array}\right)
$$

and the matrix-integral operators

$$
\begin{aligned}
K_{1} & =\partial_{x}^{-1} U_{1 x}=\int_{0}^{x} d x\left(\begin{array}{ll}
u_{x} & \alpha^{2}(\rho) \rho_{x} \\
\rho_{x} & \beta^{2}(u) u_{x}
\end{array}\right) \\
& =\int_{(0,0)}^{(u, \rho)} U_{1 u} d u+U_{1 \rho} d \rho=\partial_{u}^{-1}\left(\begin{array}{cc}
1 & 0 \\
0 & \beta^{2}(u)
\end{array}\right)+\partial_{\rho}^{-1}\left(\begin{array}{cc}
0 & \alpha^{2}(\rho) \\
1 & 0
\end{array}\right), \\
K_{2} & =\partial_{x}^{-1} U_{2 x}=\int_{0}^{x} d x\left(\begin{array}{cc}
\beta^{2}(u) u_{x} & \alpha^{2}(\rho) \rho_{x} \\
\rho_{x} & u_{x}
\end{array}\right) \\
& =\int_{(0,0)}^{(u, \rho)} U_{2 u} d u+U_{2 \rho} d \rho=\partial_{u}^{-1}\left(\begin{array}{cc}
\beta^{2}(u) & 0 \\
0 & 1
\end{array}\right)+\partial_{\rho}^{-1}\left(\begin{array}{cc}
0 & \alpha^{2}(\rho) \\
1 & 0
\end{array}\right),
\end{aligned}
$$


with the operator $\partial_{x}^{-1}$ defined as in (4.18). Here again the curvilinear integrals in the second lines are independent of the integration path. Define also the operator

$$
K=K_{1} K_{2}=\partial_{x}^{-1} U_{1 x} \partial_{x}^{-1} U_{2 x}
$$

THEOREM 4.6. The separable Hamiltonians (4.12) and their combinations $H^{(N)}\left(c_{1}, c_{2}\right)$ for $N=0,1,2, \ldots$ satisfy the recursion relation

$$
\left(H_{\rho}^{(N+2)}, H_{u}^{(N+2)}\right)^{T}=K\left(H_{\rho}^{(N)}, H_{u}^{(N)}\right)^{T}, \quad H^{(N)} \stackrel{\text { def }}{=} H^{(N)}\left(c_{1}, c_{2}\right),
$$

with the recursion operator $K$ defined by (4.23) and the solutions

$$
\begin{gathered}
\left(H_{\rho}^{(2 m)}, H_{u}^{(2 m)}\right)^{T}=K^{m}\left(H_{\rho}^{(0)}, H_{u}^{(0)}\right)^{T}=K^{m}\left(c_{1}, c_{2}\right)^{T}, \quad m=0,1,2, \ldots, \\
\left(H_{\rho}^{(2 m-1)}, H_{u}^{(2 m-1)}\right)^{T}=K^{m-1}\left(H_{\rho}^{(1)}, H_{u}^{(1)}\right)^{T}=K^{m-1} U_{1}\left(c_{1}, c_{2}\right)^{T}, \quad m=1,2, \ldots
\end{gathered}
$$

Thus, by Theorem 4.6, all the Hamiltonians of the Manin's series (4.12) are generated by the recursion operator (4.23) (see [31]). The inverse recursion operator has the form

$$
\begin{aligned}
K^{-1} & =K_{2}^{-1} K_{1}^{-1}=\left(U_{2 x}\right)^{-1} D_{x}\left(U_{1 x}\right)^{-1} D_{x}, \\
\left(U_{1 x}\right)^{-1} & =\frac{1}{\beta^{2} u_{x}^{2}-\alpha^{2} \rho_{x}^{2}}\left(\begin{array}{cc}
\beta^{2} u_{x} & -\alpha^{2} \rho_{x} \\
-\rho_{x} & u_{x}
\end{array}\right), \\
\left(U_{2 x}\right)^{-1} & =\frac{1}{\beta^{2} u_{x}^{2}-\alpha^{2} \rho_{x}^{2}}\left(\begin{array}{cc}
u_{x} & -\alpha^{2} \rho_{x} \\
-\rho_{x} & \beta^{2} u_{x}
\end{array}\right) .
\end{aligned}
$$

Define the second-order matrix-differential operator

$$
L=D_{x} K^{-1} \partial_{x}^{-1}=D_{x}\left(U_{2 x}\right)^{-1} D_{x}\left(U_{1 x}\right)^{-1} .
$$

Consider Lie equations for the $N$ th-order higher symmetries of the Hamiltonian system (4.5) with the Hamiltonian $H$ and the Hamiltonian matrix $\hat{H}$ in the evolutionary form

$$
\left(\begin{array}{l}
u \\
\rho
\end{array}\right)_{\tau}=\left(\begin{array}{l}
f\left(x, t, u, \rho, u_{x}, \rho_{x}, \ldots, u_{x}^{(N)}, \rho_{x}^{(N)}\right) \\
g\left(x, t, u, \rho, u_{x}, \rho_{x}, \ldots, u_{x}^{(N)}, \rho_{x}^{(N)}\right)
\end{array}\right) \equiv\left(\begin{array}{l}
f_{n} \\
g_{n}
\end{array}\right)
$$

that implies $x_{\tau}=t_{\tau}=0$. These symmetries satisfy the determining equation

$$
\left(I D_{t}-D_{x} \hat{H}\right)\left(\begin{array}{l}
f \\
g
\end{array}\right)=\left(\begin{array}{l}
0 \\
0
\end{array}\right) \text {. }
$$

THEOREM 4.7. Any separable Hamiltonian system (4.5) possesses the second-order matrix-differential recursion operator of the form (4.27) satisfying the recursion relation

$$
L\left(f_{n}, g_{n}\right)^{T}=\left(f_{n+2}, g_{n+2}\right)^{T}, \quad n=2,3, \ldots
$$


COROLLARY 4.8. On the solution manifolds of systems (4.5),

$$
\left[I D_{t}-D_{x} \hat{H}, L\right]=0 .
$$

Thus, the Lax representation for these systems $\partial L / \partial t=[L, A]$ is obtained with the Lax pair of the Ibragimov-Shabat type $[16,17]$, where $A=-D_{x} \hat{H}$ and $L$ is defined by $(4.27)$.

COROLLARY 4.9. For the GGD equations $\beta(u)=1, U_{1}=U_{2}=U$ and the second-order recursion operator (4.27) is reduced to the squared first-order recursion operator $L_{0}$ :

$$
L=L_{0}^{2}, \quad L_{0}=D_{x}\left(U_{x}\right)^{-1} .
$$

The operator $L_{0}$ coincides with the recursion operator (3.14) transformed from the Riemann invariants $s, r$ to the separable variables $u, \rho$, and satisfies the relation

$$
L_{0}\left(f_{n}, g_{n}\right)^{T}=\left(f_{n+1}, g_{n+1}\right)^{T}, \quad n=2,3, \ldots
$$

THEOREM 4.10. For separable Hamiltonian systems (4.5) with the generic functions $\beta(u)$ and $\alpha(\rho)$, no first-order recursion operators exist and the second-order recursion operator (4.27) cannot be reduced to a square of the first-order recursion operator or to a product of different first-order operators.

\subsection{Hydrodynamic and higher symmetries}

THeOREM 4.11. Separable systems (4.5) with the Hamiltonian $H(u, \rho)$ with no explicit dependence on $t$ admit an infinite set of hydrodynamic symmetries homogeneous in derivatives with or without an explicit dependence on $x, t$ generated by the Lie equations

$$
\begin{gathered}
\left(\begin{array}{l}
u \\
\rho
\end{array}\right)_{\tau}=(x I+t \hat{H}+\hat{h})\left(\begin{array}{c}
u \\
\rho
\end{array}\right)_{x}, \\
\left(\begin{array}{l}
u \\
\rho
\end{array}\right)_{\tau}=\hat{h}\left(\begin{array}{l}
u \\
\rho
\end{array}\right)_{x} \equiv \sigma_{1} D_{x}\left(\begin{array}{l}
h_{u}(u, \rho) \\
h_{\rho}(u, \rho)
\end{array}\right),
\end{gathered}
$$

respectively.

Here $h(u, \rho)$ is an arbitrary smooth solution of (4.11), and $\hat{H}$ and $\hat{h}$ are Hamiltonian matrices of the form (4.4).

COROLLARY 4.12. All the hydrodynamic symmetries (4.35) with no explicit dependence on $x, t$ are separable Hamiltonian systems with the Hamiltonians $h(u, \rho)$ which mutually commute and also commute with the Hamiltonian $H(u, \rho)$ of system (4.5).

Thus, any separable Hamiltonian system (4.5) is included into the infinite hierarchy of commuting separable Hamiltonian flows.

THEOREM 4.13. Hydrodynamic symmetries (4.35) of system (4.5) with no explicit dependence on $x, t$ form an invariant subspace for the second-order recursion operator $L$. This operator generates the recursion relation for the hydrodynamic symmetries

$$
L \hat{h}\left(\begin{array}{c}
u \\
\rho
\end{array}\right)_{x}=\sigma_{1} D_{x}\left(\begin{array}{l}
h_{1 u}(u, \rho) \\
h_{1 \rho}(u, \rho)
\end{array}\right) \equiv \hat{h}_{1}\left(\begin{array}{c}
u \\
\rho
\end{array}\right)_{x},
$$


and generates the recursion of their Hamiltonians

$$
h_{1}(u, \rho)=\frac{1}{\beta^{2}(u)} h_{u u}(u, \rho)=\frac{1}{\alpha^{2}(\rho)} h_{\rho \rho}(u, \rho)
$$

COROLLARY 4.14. Hydrodynamic symmetries (4.35) of the separable Hamiltonian system (4.5) with no explicit dependence on $x, t$ form an infinite-dimensional commutative Lie algebra. Its elements depend on arbitrary smooth solutions $h(u, \rho)$ of (4.11).

Denote by $h_{m}(u, \rho)$ the result of the $m$-fold application of (4.37) to $h(u, \rho)$ :

$$
h_{m}(u, \rho)=\left(\frac{\beta^{-2}(u) \partial^{2}}{\partial u^{2}}\right)^{m} h(u, \rho)=\left(\frac{\alpha^{-2}(\rho) \partial^{2}}{\partial \rho^{2}}\right)^{m} h(u, \rho) .
$$

Then formula (4.36) gives the result

$$
L^{m} \hat{h}\left(\begin{array}{c}
u \\
\rho
\end{array}\right)_{x}=\hat{h}_{m}\left(\begin{array}{c}
u \\
\rho
\end{array}\right)_{x}
$$

THEOREM 4.15. For any separable Hamiltonian system (4.5), all its higher symmetries of an even order $n=2 m$ generated by the action of the recursion operators $L^{m}$ on the hydrodynamic symmetries (4.34) are given by the formula

$$
\left(\begin{array}{l}
f_{2 m} \\
g_{2 m}
\end{array}\right)=L^{m} x\left(\begin{array}{c}
u \\
\rho
\end{array}\right)_{x}+\left(t \hat{H}_{m}+\hat{h}_{m}\right)\left(\begin{array}{c}
u \\
\rho
\end{array}\right)_{x}
$$

for $m=1,2, \ldots$

Here $H_{m}(u, \rho)$ is obtained by transformation (4.38) out of $H(u, \rho)$. For $m=1$, this formula gives all second-order symmetries of (4.5).

THEOREM 4.16. The separable system (4.5) with the Hamiltonian $H(u, \rho)$ possesses an infinite series of higher symmetries of even orders $2 m$ with no explicit dependence on $t, x$ if for some integer $N$, the Hamiltonian $H$ satisfies the condition

$$
L^{N} \hat{H}\left(\begin{array}{l}
u \\
\rho
\end{array}\right)_{x}=\left(\begin{array}{l}
0 \\
0
\end{array}\right) \Longleftrightarrow \hat{H}_{N}=0
$$

that is, the vector $\hat{H}\left(u_{x}, \rho_{x}\right)^{T}$ belongs to the kernel of the operator $L^{N}$, and then $m \geq N$.

The symmetries in Theorem 4.16 are determined by (4.40) with $\hat{H}_{m}=0$ being common symmetries of all systems (4.5) with the right-hand side belonging to the kernel of the operator $L^{N}$. For $\hat{h}_{m}=0$, the special form of higher symmetries satisfying (4.41) with $m \geq N$ is

$$
\left(\begin{array}{c}
u \\
\rho
\end{array}\right)_{\tau}=L^{m} x\left(\begin{array}{c}
u \\
\rho
\end{array}\right)_{x}=\left(\begin{array}{l}
f_{2 m}\left(u, \rho, u_{x}, \rho_{x}, \ldots, u_{x}^{(2 m)}, \rho_{x}^{(2 m)}\right) \\
g_{2 m}\left(u, \rho, u_{x}, \rho_{x}, \ldots, u_{x}^{(2 m)}, \rho_{x}^{(2 m)}\right)
\end{array}\right)
$$


For $\beta(u)=1, L=L_{0}^{2}$, where $L_{0}$ is the first-order recursion operator (4.32). Then (4.5) form the GGD system with the higher symmetries of any order $n \geq N \geq 2$ :

$$
\left(\begin{array}{l}
u \\
\rho
\end{array}\right)_{\tau}=L_{0}^{n} x\left(\begin{array}{l}
u \\
\rho
\end{array}\right)_{x}=\left(\begin{array}{l}
f_{n} \\
g_{n}
\end{array}\right)
$$

if the following condition is satisfied:

$$
L_{0}^{N} \hat{H}\left(\begin{array}{l}
u \\
\rho
\end{array}\right)_{x}=\left(\begin{array}{l}
0 \\
0
\end{array}\right)
$$

For the generic function $\beta(u)$, denote by $\hat{X}_{h}$ and $\hat{X}_{2 m}$ the evolutionary generators of the hydrodynamic symmetries (4.35) and of the higher symmetries (4.42), respectively.

THEOREM 4.17. Hydrodynamic and higher symmetries of separable Hamiltonian systems (4.5) with no explicit dependence on $x, t$ form the infinite-dimensional noncommutative Lie algebra

$$
\left[\hat{X}_{h}, \hat{X}_{\tilde{h}}\right]=0, \quad\left[\hat{X}_{2 m}, \hat{X}_{h}\right]=\hat{X}_{h_{m}}, \quad\left[\hat{X}_{2 m}, \hat{X}_{2 n}\right]=0,
$$

with the hydrodynamic symmetries $\hat{X}_{h}$ forming an infinite-dimensional commutative ideal.

COROLlary 4.18. Higher and hydrodynamic symmetries in (4.45) commute if and only if $\hat{h}_{m}=0$, where $h(u, \rho)$ are the Hamiltonians of the hydrodynamic symmetries (4.35).

Theorem 4.16 poses a problem of obtaining the kernel of the operator $L^{N}$ to solve (4.41). In order to obtain its general solution, we have to allow an explicit dependence of the Hamiltonians on $t$ and use the Manin's series (4.12) of Hamiltonians $H^{(N)}(1-v, v)$ with $v=0,1$. Let $a_{i}(t)$ denote arbitrary smooth functions and

$$
\begin{aligned}
& H^{[N]}(u, \rho, t) \\
& \begin{array}{l}
\sum_{k=0}^{N}\left[a_{4(N-k)+1}(t) H^{(2 k)}(1,0)+a_{4(N-k)+2}(t) H^{(2 k)}(0,1)\right. \\
\left.\quad+a_{4(N-k)+3}(t) H^{(2 k-1)}(1,0)+a_{4(N-k)+4}(t) H^{(2 k-1)}(0,1)\right], \quad N=1,2, \ldots . .
\end{array}
\end{aligned}
$$

Consider the explicitly $t$-dependent Hamiltonian system (4.5) with the Hamiltonians $H^{[N]}$ :

$$
\left(\begin{array}{c}
u \\
\rho
\end{array}\right)_{t}=\sigma_{1} D_{x}\left(\begin{array}{l}
H_{u}^{[N]}(u, \rho, t) \\
H_{\rho}^{[N]}(u, \rho, t)
\end{array}\right) \equiv \hat{H}^{[N]}(u, \rho, t)\left(\begin{array}{c}
u \\
\rho
\end{array}\right)_{x}, \quad N=1,2, \ldots
$$

THEOREM 4.19. The kernel of the operator $L^{N}$ coincides with the right-hand side of the Hamiltonian system (4.47):

$$
L^{-N}\left(\begin{array}{l}
0 \\
0
\end{array}\right)=\hat{H}^{[N]}(u, \rho, t)\left(\begin{array}{l}
u \\
\rho
\end{array}\right)_{x}, \quad L^{-1} \stackrel{\text { def }}{=} U_{1 x} D_{x}^{-1} U_{2 x} D_{x}^{-1} .
$$


COROLlary 4.20. Let $N$ be some integer. Any separable Hamiltonian system which has higher symmetries (4.40) with $\hat{H}_{m}=0$ (with no explicit dependence on $t, x$ ) of the order $2 m \geq 2 N$ has the form (4.47). In particular, such a system has higher symmetries (4.42) which satisfy the condition $m \geq N$.

THEOREM 4.21. A separable Hamiltonian system (4.5) with a Hamiltonian $H(u, \rho, t)$ explicitly dependent on $t$ admits an infinite set of the hydrodynamic symmetries

$$
\left(\begin{array}{c}
u \\
\rho
\end{array}\right)_{\tau}=\left[x I+\int_{0}^{t} \hat{H}(u, \rho, t) d t+\hat{h}(u, \rho)\right]\left(\begin{array}{l}
u \\
\rho
\end{array}\right)_{x}
$$

explicitly dependent on $t, x$ and on an arbitrary smooth solution $h(u, \rho)$ of the wave equation (4.11). An infinite set of higher symmetries of any even order $2 m$ for such a system is generated by the action of the recursion operators $L^{m}$ on the symmetries (4.49):

$$
\left(\begin{array}{c}
u \\
\rho
\end{array}\right)_{\tau}=L^{m} x\left(\begin{array}{c}
u \\
\rho
\end{array}\right)_{x}+\left[\int_{0}^{t} \hat{H}_{m}(u, \rho, t) d t+\hat{h}(u, \rho)\right]\left(\begin{array}{l}
u \\
\rho
\end{array}\right)_{x}
$$

and has the same extent of arbitrariness as the symmetries (4.49). In formulas (4.49) and (4.50), integrations with respect to $t$ are performed at constant values of $u, \rho$.

4.5. Invariant solutions and linearization. Consider solutions of the Hamiltonian systems (4.47) invariant with respect to the higher symmetries (4.50) with no explicit dependence on $t, x$ due to the condition $\hat{H}_{m}^{[N]}(u, \rho, t)=0$ for $m \geq N$. Invariance conditions for these solutions have the form

$$
L^{m} x\left(\begin{array}{l}
u \\
\rho
\end{array}\right)_{x}+\hat{h}(u, \rho)\left(\begin{array}{l}
u \\
\rho
\end{array}\right)_{x}=\left(\begin{array}{l}
0 \\
0
\end{array}\right), \quad m=N, N+1, \ldots,
$$

with $h(u, \rho)$ satisfying (4.11). We will obtain such solutions by the method which uses the Lax representation (4.31) and the inverse recursion operator (4.48) [30, 31]. Define the Hamiltonian $H^{[m]}(u, \rho)$ by (4.46) with $N=m$ and $a_{i}(t)=c_{i}=$ const:

$$
\begin{aligned}
H^{[m]}(u, \rho)=\sum_{k=0}^{m}[ & c_{4(m-k)+1} H^{(2 k)}(1,0)+c_{4(m-k)+2} H^{(2 k)}(0,1) \\
& \left.+c_{4(m-k)+3} H^{(2 k-1)}(1,0)+c_{4(m-k)+4} H^{(2 k-1)}(0,1)\right] .
\end{aligned}
$$

Let $H(u, \rho)$ denote an arbitrary solution of the wave equation (4.11).

THEOREM 4.22. Invariant solutions $(u(x, t), \rho(x, t))$ of system (4.47) with the Hamiltonian $H^{[N]}(u, \rho, t)$ satisfying the invariance condition (4.51) are determined by

$$
\left(\begin{array}{l}
H_{\rho u}(u, \rho) \\
H_{u u}(u, \rho)
\end{array}\right)=\left(\begin{array}{l}
x \\
0
\end{array}\right)+\int_{0}^{t}\left(\begin{array}{l}
H_{\rho u}^{[N]}(u, \rho, t) \\
H_{u u}^{[N]}(u, \rho, t)
\end{array}\right) d t
$$

if the conditions of the implicit function theorem are satisfied for (4.53).

Equation (4.53) determines the linearizing transformation which reduces the solution of the nonlinear separable Hamiltonian system (4.47) which may explicitly depend on $t$ to the solution of the linear wave equation (4.11) for $H(u, \rho)$. 
COROLLARY 4.23. The formula

$$
\left(\begin{array}{l}
H_{\rho u}^{[m]}(u, \rho) \\
H_{\mathcal{u} u}^{[m]}(u, \rho)
\end{array}\right)=\left(\begin{array}{c}
x \\
0
\end{array}\right)+\int_{0}^{t}\left(\begin{array}{l}
H_{\rho u}^{[N]}(u, \rho, t) \\
H_{\mathcal{u} u}^{[N]}(u, \rho, t)
\end{array}\right) d t, \quad m=N, N+1, \ldots,
$$

gives an infinite discrete set of exact solutions of (4.47) satisfying condition (4.51) with $h(u, \rho)=0$, invariant with respect to the higher symmetries (4.42).

For an explicit form of these solutions, expression (4.52) for $H^{[m]}(u, \rho)$ must be used.

Corollary 4.24. For system (4.47) with the Hamiltonian $H^{[N]}(u, \rho)$ having no explicit dependence on $t$, formula (4.53) gives the hodograph transformation

$$
\left(\begin{array}{l}
H_{\rho u}(u, \rho) \\
H_{u u}(u, \rho)
\end{array}\right)=\left(\begin{array}{l}
x \\
0
\end{array}\right)+t\left(\begin{array}{l}
H_{\rho u}^{[N]}(u, \rho) \\
H_{u u}^{[N]}(u, \rho)
\end{array}\right)
$$

which interchanges the roles of independent and dependent variables $(x, t)$ and $(u, \rho)$.

REMARK 4.25. Explicitly $t$-dependent Hamiltonian systems (4.47) are not linearizable by the usual hodograph transformation (4.55). The correct linearizing transformation is given by (4.53) and presents a generalization of the hodograph transformation for explicitly time-dependent systems.

THEOREM 4.26. Consider a separable system of the form (4.5) with the explicitly $t$ dependent Hamiltonian $H(u, \rho, t)$ satisfying (4.11). Its invariant solutions with respect to the hydrodynamic symmetries (4.49) are given by the equations

$$
x+\int_{0}^{t} H_{\rho u}(u, \rho, t) d t=h_{\rho u}(u, \rho), \quad \int_{0}^{t} H_{u u}(u, \rho, t) d t=h_{u u}(u, \rho),
$$

where $h(u, \rho)$ is an arbitrary smooth solution of the linear equation (4.11).

In (4.56), $H_{u u}$ and $h_{u u}$ may be replaced by $H_{\rho \rho}$ and $h_{\rho \rho}$. These formulas reduce the solution of any separable time-dependent system (4.5) to the solution of the linear equation (4.11) for $h(u, \rho)$. This is the linearizing transformation which generalizes the hodograph transformation for the explicitly time-dependent separable Hamiltonian systems (4.5).

\section{Semi-Hamiltonian equations}

5.1. Geometry of semi-Hamiltonian systems and hydrodynamic symmetries. Consider first-order quasilinear nondegenerate systems homogeneous in derivatives

$$
u_{t}^{i}=v_{i}(u) u_{x}^{i}, \quad v_{i}(u) \neq v_{j}(u) \quad(i \neq j), i=1,2, \ldots, n
$$

with a diagonal $n \times n$ matrix $V(u)=\operatorname{diag}\left(v_{i}(u)\right)$. From now on, no summation on repeated indices is assumed. Here $u=\left(u^{1}, u^{2}, \ldots, u^{n}\right)$ is $n$-vector, $u^{i}$ are Riemann invariants [27]. Higher symmetries of the $N$ th-order of system (5.1) are generated by the 
Lie equations

$$
u_{\tau}^{i}=\eta_{i}\left(x, t, u, u_{x}, \ldots, u_{x}^{(N)}\right), \quad i=1,2, \ldots, n,
$$

where $\tau$ is the group parameter and we assume that $x_{\tau}=t_{\tau}=0$. Denote $\eta=\left(\eta_{1}, \ldots, \eta_{n}\right)^{T}$.

Consider the operators $D_{x}, D_{t}$ of the total derivatives with respect to $x, t$ :

$$
\begin{aligned}
& D_{x}=\frac{\partial}{\partial x}+\sum_{j=1}^{n}\left(u_{x}^{j} \frac{\partial}{\partial u^{j}}+\sum_{k=1}^{\infty} u_{x}^{j(k+1)} \frac{\partial}{\partial u_{x}^{j(k)}}\right), \\
& D_{t}=\frac{\partial}{\partial t}+\sum_{j=1}^{n}\left(v_{j} u_{x}^{j} \frac{\partial}{\partial u^{j}}+\sum_{k=1}^{\infty} D_{x}^{k}\left[v_{j} u_{x}^{j}\right] \frac{\partial}{\partial u_{x}^{j(k)}}\right),
\end{aligned}
$$

where (5.1) is used in $D_{t}$, and $\partial / \partial x$ and $\partial / \partial t$ are taken at constant values of $u$ and $u_{x}^{(k)}$.

The compatibility conditions $u_{\tau t}^{i}=u_{t \tau}^{i}$ of systems (5.1) and (5.2) take the form of the determining equation for the symmetry characteristic $\eta$ :

$$
\left(I D_{t}-V D_{x}-U_{x}\left(\frac{\partial V}{\partial U}\right)\right)[\eta]=0
$$

Here $I$ is the unit matrix, $U_{x}=\operatorname{diag}\left(u_{x}^{i}\right)$, and $\partial V / \partial U=\left(v_{i, u^{j}}\right)$ is the Jacobian matrix.

For the hydrodynamic symmetries, we choose $N=1$ in the Lie equations (5.2). Here we consider only hydrodynamic symmetries linear homogeneous in derivatives

$$
u_{\tau}^{i}=W_{i}(u, x, t) u_{x}^{i}, \quad i=1,2, \ldots, n .
$$

Define the symmetrical connection coefficients associated with system (5.1) [39]:

$$
\begin{gathered}
\Gamma_{i j}^{i}(u)=\Gamma_{j i}^{j}(u)=\frac{v_{i, u^{j}}(u)}{\left(v_{j}-v_{i}\right)} \quad(i \neq j), \\
\Gamma_{i i}^{j}=-\left(\frac{g_{i i}}{g_{j j}}\right) \Gamma_{i j}^{i} \quad(i \neq j), \quad \Gamma_{j k}^{i}=0 \quad(i \neq j \neq k \neq i) .
\end{gathered}
$$

This connection is compatible with the nondegenerate diagonal metric

$$
g_{i i}(u)=H_{i}^{2}(u)=e^{2 \Phi_{i}(u)}, \quad g_{i j}=0 \quad(i \neq j), \quad \operatorname{det}\left(g^{i j}\right) \neq 0 .
$$

Here $H_{i}(u)$ are Lamé coefficients. The connection is determined by the metric

$$
\Gamma_{i j}^{i}=\left(\ln \sqrt{g_{i i}}\right)_{u^{j}}=\left(\ln H_{i}\right)_{u^{j}}=\Phi_{i, u^{j}}(u) .
$$

Integrability conditions $\Gamma_{i j, u^{k}}^{i}=\Gamma_{i k, u^{j}}^{i}$ of system (5.9) with the use of the expressions (5.6) take the form of Tsarëv's conditions [39]:

$$
\left[\frac{v_{i, u^{j}}}{\left(v_{j}-v_{i}\right)}\right]_{u^{k}}=\left[\frac{v_{i, u^{k}}}{\left(v_{k}-v_{i}\right)}\right]_{u^{j}} \quad(i \neq j \neq k \neq i) .
$$

DEFINITION 5.1 (see [40]). Diagonal nondegenerate system (5.1) is called semiHamiltonian if its coefficients $v_{i}(u)$ satisfy conditions (5.10).

We will consider the generic case excluding weakly nonlinear systems [27] $\left(v_{i, u^{i}}=0\right.$ for all $i$ ) which admit more general symmetries than in the following theorem. 
THEOREM 5.2 (see [40]). The generic diagonal system (5.1) admits an infinite set of hydrodynamic symmetries with no explicit dependence on $x, t$

$$
u_{\tau}^{i}=w_{i}(u) u_{x}^{i}, \quad i=1,2, \ldots, n,
$$

with a functional arbitrariness if and only if (5.1) is semi-Hamiltonian, that is, condition (5.10) is satisfied.

The symmetries in Theorem 5.2 are generated by Lie equations (5.11) with the coefficients $w_{i}(u)$ which form an arbitrary smooth solution of the linear system

$$
w_{i, u^{j}}=\Gamma_{i j}^{i}\left(w_{j}-w_{i}\right) \quad(i \neq j)
$$

with $\Gamma_{i j}^{i}(u)$ defined by (5.6). All these symmetries mutually commute. The set of such symmetries is locally parameterized by $n$ arbitrary functions $c_{i}\left(u^{i}\right)$ of one variable.

THEOREM 5.3 (see [33]). Semi-Hamiltonian systems of the form (5.1) admit an infinite set of hydrodynamic symmetries explicitly dependent on $x, t$ with the same functional arbitrariness as in Theorem 5.2.

The symmetries in Theorem 5.3 are generated by the Lie equations

$$
u_{\tau}^{i}=\left[w_{i}(u)+c\left(x+t v_{i}(u)\right)\right] u_{x}^{i}, \quad i=1,2, \ldots, n,
$$

where $w_{i}(u)$ is an arbitrary solution of the linear system (5.12) and $c$ is an arbitrary constant.

THEOREM 5.4 (see [40]). Any semi-Hamiltonian system of the form (5.1) with the nondegenerate metric (5.8) is a Hamiltonian system if and only if the following components of the Riemann curvature tensor vanish: $R_{j j i}^{i}=0(i \neq j)$. Then the curvature tensor vanishes identically and the variables $u^{i}$ form an orthogonal curvilinear coordinate system in a flat (pseudo-Euclidean) space.

COROLlARY 5.5. For the semi-Hamiltonian system (5.1), the following components of the curvature tensor vanish for $i \neq j \neq k \neq l$ and $i \neq j \neq k \neq i$ :

$$
\begin{gathered}
R_{j k l}^{i}=0, \quad R_{i k j}^{i} \equiv \Gamma_{i j, u^{k}}^{i}-\Gamma_{i k, u^{j}}^{i}=0, \\
R_{j k i}^{i} \equiv \Gamma_{i j, u^{k}}^{i}-\left[\Gamma_{i k}^{i} \Gamma_{k j}^{k}+\Gamma_{i j}^{i}\left(\Gamma_{j k}^{j}-\Gamma_{i k}^{i}\right)\right]=0 .
\end{gathered}
$$

REMARK 5.6. These equations give compatibility conditions for the linear system (5.12) and they are equivalent to the Tsarëv's conditions (5.10).

5.2. Linearization and invariant solutions of semi-Hamiltonian systems. Consider hydrodynamic symmetries (5.13) explicitly dependent on $x, t$ with $c=-1$. Then invariant solutions of the semi-Hamiltonian system (5.1) with $u_{x} \neq 0$ are determined by

$$
w_{i}(u)=t v_{i}(u)+x, \quad i=1,2, \ldots, n .
$$

THEOREM 5.7 (see [40]). Let $w_{i}(u)$ in (5.15) form an arbitrary smooth solution of the linear system (5.12). Then any smooth solution $u^{i}(x, t)$ of (5.15) is a solution of the 
semi-Hamiltonian system (5.1). Vice versa, any solution $u^{i}(x, t)$ of $(5.1)$ is a local solution of (5.15) in the vicinity of such a point $\left(x_{0}, t_{0}\right)$, where $u_{x}^{i}\left(x_{0}, t_{0}\right) \neq 0$ for each value of $i$.

REMARK 5.8. Equations (5.15) determine a linearizing transformation for the semiHamiltonian system (5.1), that is, to obtain explicit formulas for the invariant solutions of the nonlinear system (5.1), one must solve the linear system (5.12) for $w_{i}(u)$ with variable coefficients. It generalizes the classical hodograph transformation for the case of multicomponent systems and it is called the generalized hodograph transformation [40]. We discover the group-theoretical origin of the linearizing transformation: every nonsingular solution of system (5.1) is an invariant solution with respect to hydrodynamic symmetries (5.13) and the extent of arbitrariness of symmetries and invariant solutions is the same as for the general solution of (5.1), that is, $n$ arbitrary functions $c_{i}\left(u^{i}\right)$ of one variable.

5.3. First-order recursion operators. By definition, recursion operator $R$ maps any symmetry of the semi-Hamiltonian system (5.1) again into its symmetry, that is, any solution $\eta=\left(\eta_{1}, \ldots, \eta_{n}\right)^{T}$ of the determining equation (5.4) is mapped again into the solution $R[\eta]$. For this to be true, it is sufficient for the operator $R$ to commute with the operator of the determining equation

$$
\left[I D_{t}-V D_{x}-U_{x}\left(\frac{\partial V}{\partial U}\right), R\right]=0
$$

on solution manifolds of (5.1) and (5.4). For systems (5.1), we use a different form of recursion operators than (2.48). In particular, for the first-order recursion operators,

$$
R=\left(A D_{x}+B\right) U_{x}^{-1}
$$

where $A=A(u)$ and $B=B\left(u, u_{x}\right)$ are $n \times n$ matrices. Define the functions

$$
S_{i}(u)=\sum_{k=1}^{n} \Gamma_{i k}^{i}(u) c_{k}\left(u^{k}\right)+d_{i}\left(u^{i}\right), \quad i=1,2, \ldots, n,
$$

which depend on $2 n$ functions $c_{i}\left(u^{i}\right)$ and $d_{i}\left(u^{i}\right)$ of one variable.

THEOREM 5.9 (see [38]). For semi-Hamiltonian systems (5.1), there exists a first-order recursion operator $R$ of the form (5.17) if and only if there exist $2 n$ functions $c_{i}\left(u^{i}\right)$ and $d_{i}\left(u^{i}\right)$ of one variable which satisfy the conditions

$$
S_{i, u^{j}}(u)=\Gamma_{i j}^{i}\left(S_{j}-S_{i}\right) \quad(i \neq j)
$$

with $S_{i}(u)$ defined by (5.18).

Matrix elements of the recursion operator in Theorem 5.9 are

$$
\begin{aligned}
R_{i j}= & {\left[\delta_{i j} c_{j}\left(u^{j}\right)\left(D_{x}+\sum_{k=1}^{n} \Gamma_{i k}^{i}(u) u_{x}^{k}\right)+\Gamma_{i j}^{i}(u)\left(c_{j}\left(u^{j}\right) u_{x}^{i}-c_{i}\left(u^{i}\right) u_{x}^{j}\right)\right]\left(\frac{1}{u_{x}^{j}}\right) } \\
& +d_{j}\left(u^{j}\right) \delta_{i j},
\end{aligned}
$$

where $\delta_{i j}$ is the Kroneker symbol. 
THEOREM 5.10. For semi-Hamiltonian systems (5.1), hydrodynamic symmetries (5.11) homogeneous in derivatives with no explicit dependence on $x, t$, subject to condition (5.12), form an invariant subspace for the recursion operator (5.20).

A restriction of the operator $R$ to the invariant subspace becomes

$$
R\left(\begin{array}{c}
w_{1}(u) u_{x}^{1} \\
\vdots \\
w_{n}(u) u_{x}^{n}
\end{array}\right)=\left(\begin{array}{c}
\hat{w}_{1}(u) u_{x}^{1} \\
\vdots \\
\hat{w}_{n}(u) u_{x}^{n}
\end{array}\right)
$$

where the functions $\hat{w}_{i}(u)$ are determined by the formula

$$
\hat{w}_{i}(u)=c_{i}\left(u^{i}\right) w_{i, u^{i}}(u)+d_{i}\left(u^{i}\right) w_{i}(u)+\sum_{k=1}^{n} \Gamma_{i k}^{i}(u) c_{k}\left(u^{k}\right) w_{k}(u)
$$

COROLLARY 5.11. For any solution $\left\{w_{i}(u)\right\}$ of the linear system (5.12), the functions $\hat{w}_{i}(u)$ also form a solution of this system if and only if the conditions (5.19) are satisfied. Then (5.22) defines a recursion for solutions of system (5.12).

The first-order recursion operator (5.20) for the multicomponent system (5.1) was constructed originally by a straightforward solution of (5.16) [38]. A new simpler method for constructing recursion operators based on the study of symmetries of the set of hydrodynamic symmetries of system (5.1) was developed by the author in [35], where the group-theoretical origin of recursion operators was discovered. A geometrical sense of the existence conditions (5.19) was also clarified in this paper.

To be explicit, consider other orthogonal curvilinear coordinates $\left\{r^{i}\right\}$, which will be specified later, with the Lamé coefficients $H_{i}(r)=\sqrt{g_{i i}(r)}=e^{\Phi_{i}(r)}$. Consider the rotation coefficients $\beta_{j i}(r)$ of this coordinate system defined by the equations $[6,40]$

$$
H_{i, r^{j}}=\beta_{j i} H_{j} \quad(i \neq j)
$$

Let $\rho=\left\{\left(r^{k}-r^{l}\right)\right\}$ denote the set of the coordinate differences.

THEOREM 5.12 (see [35]). First-order recursion operator for the semi-Hamiltonian system (5.1) exists if and only if the rotation coefficients $\beta_{j i}(r)$ of some curvilinear orthogonal coordinate system $r^{i}$ depend only on the coordinate differences $\rho: \beta_{j i}=\beta_{j i}(\rho)$.

5.4. Second-order recursion operators. We will consider second-order recursion operators in the form

$$
R=\left(A D_{x}^{2}+B D_{x}+F\right) U_{x}^{-1},
$$

where $A=A\left(u, u_{x}\right), B=B\left(u, u_{x}, u_{x x}\right)$, and $F=F\left(u, u_{x}, u_{x x}, u_{x x x}\right)$ are $n \times n$ matrices. Define the "connection potential" $V(u)$ by a completely integrable system (in the sense 
of Frobenius)

$$
V_{u^{i} u^{j}}(u)=\Gamma_{i j}^{i} \Gamma_{j i}^{j} \quad(i \neq j)
$$

so that $V(u)$ depends on $n$ arbitrary functions of one variable. Integrability conditions for (5.25) are satisfied as a consequence of the property (5.10). Define

$$
\begin{aligned}
& b_{i k}(u)=f_{k}\left(u^{k}\right)\left[\Gamma_{i k}^{i}\left(2 \Gamma_{k k}^{k}-\Gamma_{i k}^{i}\right)-\Gamma_{i k, u^{k}}^{i}\right]+\left[c_{k}\left(u^{k}\right)-f_{k}^{\prime}\left(u^{k}\right)\right] \Gamma_{i k}^{i} \quad(i \neq k), \\
& b_{i i}(u)=f_{i}\left(u^{i}\right)\left[\Gamma_{i i, u^{i}}^{i}+\left(\Gamma_{i i}^{i}\right)^{2}-2 V_{u^{i} u^{i}}(u)\right]-f_{i}^{\prime}\left(u^{i}\right) V_{u^{i}}(u)+c_{i}\left(u^{i}\right) \Gamma_{i i}^{i}+d_{i}\left(u^{i}\right),
\end{aligned}
$$

and $B_{i}(u)=\sum_{k=1}^{n} b_{i k}$ which depend on $3 n$ functions $f_{i}\left(u^{i}\right), c_{i}\left(u^{i}\right)$, and $d_{i}\left(u^{i}\right)$ of one variable. Let $R_{i k}, A_{i k}, B_{i k}$, and $F_{i k}$ denote matrix elements of the operator $R$ and of the matrices $A, B$, and $F$, respectively.

DEFINITION 5.13. Let $H_{i}(u)$ and $\beta_{i j}(u)$ denote Lamé and rotation coefficients, respectively, of the curvilinear orthogonal system $\left\{u^{i}\right\}$, and let $G_{i}\left(\hat{u}^{i}\right)$ denote a function of $u$ independent of $u^{i}$. Semi-Hamiltonian system (5.1) is called generic (with respect to second-order recursion operators) if none of the following cases occurs:

$$
\begin{gathered}
v_{i, u^{i}}(u)=0, \quad v_{i, u^{i}}(u)=\left(\frac{F_{i}\left(u^{i}\right) G_{i}\left(\hat{u}^{i}\right)}{g_{i i}}\right) e^{V(u)}, \\
{\left[\frac{\beta_{j i}}{\left(v_{j}-v_{i}\right)}\right]\left[\frac{\left(\beta_{k i} / H_{i}\right)_{u^{i}}}{v_{i, u^{i}}}\right]_{u^{i}}=\left[\frac{\beta_{k i}}{\left(v_{k}-v_{i}\right)}\right]\left[\frac{\left(\beta_{j i} / H_{i}\right)_{u^{i}}}{v_{i, u^{i}}}\right]_{u^{i}} \quad(i \neq j \neq k) .}
\end{gathered}
$$

THEOREM 5.14 (see [35]). For the semi-Hamiltonian generic system (5.1), the secondorder recursion operator $R$ of the form (5.24) exists if and only if there exist $3 n$ functions $f_{i}\left(u^{i}\right), c_{i}\left(u^{i}\right)$, and $d_{i}\left(u^{i}\right)$ of one variable which satisfy the conditions

$$
B_{i, u^{j}}(u)=\Gamma_{i j}^{i}\left(B_{j}-B_{i}\right) \quad(i \neq j)
$$

with the functions $B_{i}(u)$ defined by (5.26).

Its matrix elements in Theorem 5.14 are given by

$$
\begin{aligned}
R_{i k} & =\left(A_{i k} D_{x}^{2}+B_{i k} D_{x}+F_{i k}\right)\left(\frac{1}{u_{x}^{k}}\right), \\
A_{i k} & =\frac{\delta_{i k} f_{i}\left(u^{i}\right)}{u_{x}^{i}}, \\
B_{i k} & =\Gamma_{i k}^{i}\left[f_{k}\left(u^{k}\right)\left(\frac{u_{x}^{i}}{u_{x}^{k}}\right)-f_{i}\left(u^{i}\right)\left(\frac{u_{x}^{k}}{u_{x}^{i}}\right)\right] \quad(i \neq k), \\
B_{i i} & =\frac{-f_{i}\left(u^{i}\right) u_{x x}^{i}}{\left[\left(u_{x}^{i}\right)^{2}\right]}+2\left[\frac{f_{i}\left(u^{i}\right)}{u_{x}^{i}}\right] \sum_{j \neq i} \Gamma_{i j}^{i} u_{x}^{j}+2 f_{i}\left(u^{i}\right) \Gamma_{i i}^{i}+c_{i}\left(u^{i}\right),
\end{aligned}
$$




$$
\begin{aligned}
F_{i k}= & f_{i}\left(u^{i}\right) \Gamma_{i k}^{i}\left(\frac{u_{x}^{k}}{u_{x}^{i}}\right)\left[\frac{u_{x x}^{i}}{u_{x}^{i}}-\frac{u_{x x}^{k}}{u_{x}^{k}}\right] \\
& +f_{k}\left(u^{k}\right)\left[\frac{\left(u_{x}^{i}\right)^{2}}{u_{x}^{k}}\right] \Gamma_{i k}^{i} \Gamma_{k i}^{k}-f_{i}\left(u^{i}\right)\left[\left[\frac{\left(u_{x}^{k}\right)^{2}}{u_{x}^{i}}\right] \Gamma_{i k, u^{k}}^{i}+\left(\Gamma_{i k}^{i}\right)^{2}\right] \\
& +\left\{f_{i}\left(u^{i}\right)\left(\Gamma_{i k}^{i} \Gamma_{k i}^{k}-2 \Gamma_{i i, u^{k}}^{i}\right)-\left[2 f_{i}\left(u^{i}\right) \Gamma_{i i}^{i}+c_{i}\left(u^{i}\right)\right] \Gamma_{i k}^{i}\right\} u_{x}^{k} \\
& +b_{i k}(u) u_{x}^{i}-f_{i}\left(u^{i}\right)\left(\frac{u_{x}^{k}}{u_{x}^{i}}\right) \sum_{j \neq i, k} u_{x}^{j}\left(\Gamma_{i k}^{i} \Gamma_{k j}^{k}+\Gamma_{i j}^{i} \Gamma_{j k}^{j}\right) \\
& +u_{x}^{i} \sum_{j \neq i, k}\left[f_{k}\left(u^{k}\right)\left(\frac{u_{x}^{j}}{u_{x}^{k}}\right) \Gamma_{i k}^{i} \Gamma_{k j}^{k}-f_{j}\left(u^{j}\right)\left(\frac{u_{x}^{k}}{u_{x}^{j}}\right) \Gamma_{i j}^{i} \Gamma_{j k}^{j}\right] \quad(i \neq k), \\
F_{i i}= & B_{i}(u) u_{x}^{i}-\sum_{k \neq i} F_{i k} .
\end{aligned}
$$

TheOREm 5.15 (see [35]). For semi-Hamiltonian systems (5.1), hydrodynamic symmetries (5.11) homogeneous in derivatives, with no explicit dependence on $x, t$, subject to condition (5.12) form the invariant subspace for the second-order recursion operator (5.30).

The action of the recursion operator (5.30) on the invariant subspace is determined by (5.21) with the new definition of functions:

$$
\begin{aligned}
\hat{w}_{i}(u)= & f_{i}\left(u^{i}\right) w_{i, u^{i} u^{i}}+\left[2 f_{i}\left(u^{i}\right) \Gamma_{i i}^{i}+c_{i}\left(u^{i}\right)\right] w_{i, u^{i}} \\
& +\sum_{k \neq i} f_{k}\left(u^{k}\right) \Gamma_{i k}^{i} w_{k, u^{k}}+\sum_{k=1}^{n} b_{i k}(u) w_{k},
\end{aligned}
$$

where $b_{i k}(u)$ are defined by (5.26).

COROLlARY 5.16. For any solution $\left\{w_{i}(u)\right\}$ of the linear system (5.12), formula (5.36) again gives a solution $\left\{\hat{w}_{i}(u)\right\}$ of (5.12) if and only if conditions (5.29) are satisfied. Thus, (5.36) is a second-order recursion for solutions of system (5.12).

THEOREM 5.17 (see [35]). If the first-order recursion operator exists, then there also exists the second-order recursion operator equal to the squared first-order recursion operator. The converse is not true: the existence conditions (5.19) for the first-order recursion operator do not follow from the existence conditions (5.29) for the second-order recursion operator.

Thus, the existence conditions for the second-order recursion operator are less restrictive than for the first-order operator.

REMARK 5.18. If system (5.1) is Hamiltonian, that is, all the components of the Riemann curvature tensor vanish, $R_{j k l}^{i}=0$ (see Theorem 5.4), then the existence conditions for recursion operators and for compatible bi-Hamiltonian structures of the hydrodynamic type [7] should be related to each other due to the Magri's theorem [19]. It seems interesting to discover these relations.

5.5. Generation of infinite series of exact solutions. To obtain explicit formulas for invariant solutions of (5.1), one must know solutions of the linear system (5.12) 
and substitute these solutions for the set of functions $\left\{w_{i}(u)\right\}$ in the linearizing transformation (5.15). Existence of a recursion operator for the semi-Hamiltonian system (5.1) is the additional constraint which makes it possible to obtain particular solutions of (5.12).

The linear system (5.12) has two trivial solutions

$$
w_{i}=1, \quad w_{i}=v_{i}(u), \quad i=1,2, \ldots, n .
$$

They serve as "seed" elements for the generation of infinite series of nontrivial solutions by recursion operators. In particular, assume that the first-order recursion operator (5.20) exists for system (5.1), that is, conditions (5.19) are satisfied. It generates the recursion (5.22) for solutions of system (5.12):

$$
\begin{gathered}
\hat{w}_{i}(u)=\left(\hat{R}_{1}[w]\right)_{i}=\sum_{k=1}^{n}\left(\hat{R}_{1}\right)_{i k}\left[w_{k}\right] \\
\left(\hat{R}_{1}\right)_{i k}=\delta_{i k}\left[c_{i}\left(u^{i}\right) \frac{\partial}{\partial u^{i}}+d_{i}\left(u^{i}\right)\right]+\Gamma_{i k}^{i}(u) c_{k}\left(u^{k}\right) .
\end{gathered}
$$

The operator $\hat{R}_{1}$ maps trivial solutions (5.37) to the nontrivial solutions of (5.12):

$$
\begin{aligned}
& \hat{w}_{i}(u)=\left(\hat{R}_{1}[1]\right)_{i}=\sum_{k=1}^{n} \Gamma_{i k}^{i}(u) c_{k}\left(u^{k}\right)+d_{i}\left(u^{i}\right) \equiv S_{i}(u), \\
& \hat{w}_{i}(u)=\left(\hat{R}_{1}[v]\right)_{i}=c_{i}\left(u^{i}\right) v_{i, u^{i}}(u)+d_{i}\left(u^{i}\right) v_{i}(u)+\sum_{k=1}^{n} \Gamma_{i k}^{i}(u) c_{k}\left(u^{k}\right) v_{k}(u) .
\end{aligned}
$$

Substituting (5.39) in (5.15), we obtain solutions $u^{i}=u^{i}(x, t)$ of system (5.1):

$$
\begin{gathered}
\sum_{k=1}^{n} \Gamma_{i k}^{i}(u) c_{k}\left(u^{k}\right)+d_{i}\left(u^{i}\right)=t v_{i}(u)+x, \quad i=1, \ldots, n, \\
c_{i}\left(u^{i}\right) v_{i, u^{i}}(u)+d_{i}\left(u^{i}\right) v_{i}(u)+\sum_{k=1}^{n} \Gamma_{i k}^{i}(u) c_{k}\left(u^{k}\right) v_{k}(u)=t v_{i}(u)+x
\end{gathered}
$$

determined as implicit functions.

The action of powers of $\hat{R}_{1}$ on the trivial solutions (5.37) generates the explicit formulas for two infinite sets of invariant solutions, with $N=1,2, \ldots$,

$$
\left(\hat{R}_{1}^{N}[1]\right)_{i}=t v_{i}(u)+x, \quad\left(\hat{R}_{1}^{N}[v]\right)_{i}=t v_{i}(u)+x
$$

Assume now the less restrictive existence conditions (5.29) for the second-order recursion operator. Then there exists the recursion (5.36) for solutions of (5.12):

$$
\begin{aligned}
& \hat{w}_{i}(u)=\left(\hat{R}_{2}[w]\right)_{i}=\sum_{k=1}^{n}\left(\hat{R}_{2}\right)_{i k}\left[w_{k}\right], \\
& \left(\hat{R}_{2}\right)_{i k}=\delta_{i k}\left\{f_{i}\left(u^{i}\right) \frac{\partial^{2}}{\left(\partial u^{i}\right)^{2}}+\left[f_{i}\left(u^{i}\right) \Gamma_{i i}^{i}(u)+c_{i}\left(u^{i}\right)\right] \frac{\partial}{\partial u^{i}}\right\}+f_{k}\left(u^{k}\right) \Gamma_{i k}^{i}(u) \frac{\partial}{\partial u^{k}}+b_{i k}(u),
\end{aligned}
$$

where $\hat{R}_{2}$ is the second-order recursion operator and $b_{i k}(u)$ are defined by (5.26). 
Trivial solutions (5.37) are mapped by $\hat{R}_{2}$ to nontrivial solutions of system (5.12) and via (5.15) to the corresponding solutions of system (5.1)

$$
\begin{aligned}
\left(\hat{R}_{2}[1]\right)_{i} \equiv & \sum_{k=1}^{n} b_{i k}(u) \equiv B_{i}(u)=t v_{i}(u)+x \\
\left(\hat{R}_{2}[v]\right)_{i} \equiv & f_{i}\left(u^{i}\right) v_{i, u^{i} u^{i}}(u)+\left[2 f_{i}\left(u^{i}\right) \Gamma_{i i}^{i}(u)+c_{i}\left(u^{i}\right)\right] v_{i, u^{i}}(u) \\
& +\sum_{k \neq i} f_{k}\left(u^{k}\right) \Gamma_{i k}^{i}(u) v_{k, u^{k}}(u)+\sum_{k=1}^{n} b_{i k}(u) v_{k}(u)=t v_{i}(u)+x .
\end{aligned}
$$

The powers $\hat{R}_{2}^{N}$ generate two infinite sets of invariant solutions from (5.37)

$$
\left(\hat{R}_{2}^{N}[1]\right)_{i}=t v_{i}(u)+x, \quad\left(\hat{R}_{2}^{N}[v]\right)_{i}=t v_{i}(u)+x, \quad N=1,2, \ldots
$$

We can use in (5.15) linear combinations of solutions of the two sets for $w_{i}(u)$

$$
c_{1}\left(\hat{R}_{1,2}^{N}[1]\right)_{i}+c_{2}\left(\hat{R}_{1,2}^{M}[v]\right)_{i}=t v_{i}(u)+x, \quad N, M=1,2, \ldots,
$$

with the operator $\hat{R}_{1}$ or $\hat{R}_{2}$ and arbitrary constants $c_{1}, c_{2}$.

Further generalization is obtained if we substitute for the characteristic $\eta^{d}=x+$ $t v_{i}(u)$ of the dilatation symmetry group in formulas (5.13) and (5.15) the results of the action on $\eta^{d}$ of the operators $\hat{R}_{1}^{L}$ or $\hat{R}_{2}^{L}$, where $L$ is any positive integer. Then we obtain the formula

$$
c_{1}\left(\hat{R}_{1,2}^{N}[1]\right)_{i}+c_{2}\left(\hat{R}_{1,2}^{M}[v]\right)_{i}=t\left(\hat{R}_{1,2}^{L}[v]\right)_{i}+x\left(\hat{R}_{1,2}^{L}[1]\right)_{i}
$$

If $L>N$ or $L>M$, (5.46) is equivalent to (5.45) with negative powers of $\hat{R}_{1,2}$.

REMARK 5.19. The operators $\hat{R}_{1}$ and $\hat{R}_{2}$ coincide with the first- and second-order symmetry generators for the linear system (5.12) essential for the separation of variables in linear equations [3, 22]. Solution of $(5.12)$ by the separation of variables would mean solving completely the nonlinear system (5.1). Thus, the linearizing transformation (5.15) presents an extension of the method of separation of variables to nonlinear systems "rich in symmetries."

\subsection{Higher symmetries of semi-Hamiltonian systems}

THEOREM 5.20 (see [33]). All second-order symmetries of the semi-Hamiltonian system (5.1) are generated by the second-order recursion operator (5.30) out of the hydrodynamic symmetries (5.13) (with $c=1$ ). The corresponding Lie equations have the form

$$
\begin{aligned}
u_{\tau}^{i}= & \eta_{2, i} \equiv \sum_{j=1}^{n} R_{i j}\left[u_{x}^{j}\left(x+t v_{j}(u)+w_{j}(u)\right)\right] \equiv-\frac{f_{i}\left(u^{i}\right) u_{x x}^{i}}{\left(u_{x}^{i}\right)^{2}} \\
& +\frac{f_{i}\left(u^{i}\right)}{u_{x}^{i}} \sum_{j \neq i} \Gamma_{i j}^{i} u_{x}^{j}+u_{x}^{i} \sum_{j \neq i} \Gamma_{i j}^{i} \frac{f_{j}\left(u^{j}\right)}{u_{x}^{j}}+2 f_{i}\left(u^{i}\right) \Gamma_{i i}^{i} \\
& +c_{i}\left(u^{i}\right)+u_{x}^{i}\left[x B_{i}(u)+t\left(\hat{R}_{2}[v]\right)_{i}+\hat{w}_{i}(u)\right], \quad i=1, \ldots, n,
\end{aligned}
$$


where the set of functions $\left\{\hat{w}_{i}(u)\right\}$ is an arbitrary solution of the linear system (5.12). The existence conditions (5.29) for the second-order symmetries and for the second-order recursion operators coincide and must be satisfied by a choice of functions $f_{i}\left(u^{i}\right), c_{i}\left(u^{i}\right)$, and $d_{i}\left(u^{i}\right)$. In the case when $B_{i}(u) \neq 0$ and $\left(\hat{R}_{2}[v]\right)_{i} \neq 0$, these symmetries explicitly depend on $x, t$. The action of powers $R^{N}$ of the recursion operator with $N=1,2, \ldots$ on the same hydrodynamic symmetries generates the infinite series of higher symmetries of system (5.1):

$$
u_{\tau}^{i}=\sum_{j=1}^{n}\left(R^{N}\right)_{i j}\left[u_{x}^{j}\left(x+t v_{j}(u)+w_{j}(u)\right)\right]
$$

If $R$ is a second-order recursion operator, then all these symmetries are of an even or$\operatorname{der} 2 N$.

\section{Multicomponent diagonal systems explicitly dependent on $t$ or $x$}

6.1. Hydrodynamic symmetries of time-dependent systems. Consider first-order quasilinear diagonal explicitly $t$-dependent systems with $n \geq 3$

$$
u_{t}^{i}=v_{i}(u, t) u_{x}^{i}, \quad i=1,2, \ldots, n
$$

subject to the condition $v_{i} \neq v_{j}$ for $i \neq j$. We search for hydrodynamic symmetries of these systems with the Lie equations $x_{\tau}=t_{\tau}=0$ and

$$
u_{\tau}^{i}=\sum_{j=1}^{n} A_{j}^{i}(u, t, x) u_{x}^{j}, \quad i=1,2, \ldots, n
$$

Define the functions

$$
c_{i j}(u, t)=\frac{v_{i, u}(u, t)}{\left(v_{j}-v_{i}\right)}, \quad \Gamma_{i j}^{i}(u)=c_{i j}(u, 0) \quad(i \neq j)
$$

THEOREM 6.1 (see [13, 28, 32]). Diagonal $n$-component system (6.1) of the hydrodynamic type with an explicit time dependence admits an infinite set of hydrodynamic symmetries of the form (6.2) with a functional arbitrariness if and only if its coefficients satisfy (5.10) and the condition

$$
\left[\frac{v_{i, u^{j}}(u, t)}{v_{i}(u, t)-v_{j}(u, t)}\right]_{t}=\beta v_{i, u^{j}}(u, t)
$$

for $i \neq j$ with an arbitrary real constant $\beta$.

The symmetries in Theorem 6.1 are generated by the Lie equations

$$
u_{\tau}^{i}=A^{i}(u, t, x) u_{x}^{i}, \quad i=1,2, \ldots, n
$$


with the coefficients $A_{i}$ defined by the formulas

$$
\begin{aligned}
& A_{i}(u, t, x)=w_{i}(u) \exp \left\{\beta\left[x+\int_{0}^{t} v_{i}(u, t) d t\right]\right\}+C \text { for } \beta \neq 0, \\
& A_{i}(u, t, x)=w_{i}(u)+C\left[x+\int_{0}^{t} v_{i}(u, t) d t\right] \text { for } \beta=0,
\end{aligned}
$$

where $C$ is an arbitrary constant, the integrations with respect to $t$ are performed at a constant value of $u$, and $\left\{w_{i}(u)\right\}$ form an arbitrary smooth solution of the linear system (5.12) with $\Gamma_{i j}^{i}$ defined by (6.3). The solution manifold of (5.12) depends on $n$ arbitrary functions $c_{i}\left(u^{i}\right)$ of one variable locally parameterizing the set of hydrodynamic symmetries.

6.2. Hydrodynamic symmetries of $x$-dependent systems. Let coefficients of the diagonal system explicitly depend on the coordinate $x$ :

$$
u_{t}^{i}=\tilde{v}_{i}(u, x) u_{x}^{i}, \quad i=1,2, \ldots, n, n \geq 3,
$$

and satisfy the condition $\tilde{v}_{i} \neq \tilde{v}_{j}(i \neq j)$. Define the functions

$$
\begin{gathered}
\tilde{c}_{i j}(u, x)=\frac{\tilde{v}_{i, u^{j}}(u, x)}{\tilde{v}_{j}-\tilde{v}_{i}}, \\
\Gamma_{i j}^{i}(u)=\frac{\tilde{c}_{i j}(u, 0) \tilde{v}_{j}(u, 0)}{\tilde{v}_{i}(u, 0)} \quad(i \neq j) .
\end{gathered}
$$

THEOREM 6.2 (see [13, 28, 32]). Diagonal n-component systems (6.7) of the hydrodynamic type with an explicit $x$-dependence admit an infinite set of hydrodynamic symmetries of the form (6.2) with a functional arbitrariness if and only if its coefficients satisfy (5.10) with the change of $\tilde{v}_{i}$ to $v_{i}$ and the condition

$$
\left[\frac{\left(\tilde{v}_{i}^{-1}(u, x)\right)_{u^{j}}}{\tilde{v}_{i}^{-1}(u, x)-\tilde{v}_{j}^{-1}(u, x)}\right]_{x}=\beta\left(\tilde{v}_{i}^{-1}(u, x)\right)_{u^{j}} \quad(i \neq j)
$$

with an arbitrary real constant $\beta$.

The symmetries in Theorem 6.2 are generated by the Lie equations

$$
u_{\tau}^{i}=\tilde{A}_{i}(u, t, x) u_{x}^{i}, \quad i=1,2, \ldots, n,
$$

with the coefficients $\tilde{A}_{i}$ defined by the formulas

$$
\begin{array}{ll}
\tilde{A}_{i}(u, t, x)=\tilde{v}_{i}(u, x)\left\{w_{i}(u) \exp \left[\beta\left(t+\int_{0}^{x} \tilde{v}_{i}^{-1}(u, x) d x\right)\right]+C\right\} & \text { for } \beta \neq 0, \\
\tilde{A}_{i}(u, t, x)=\tilde{v}_{i}(u, x)\left\{w_{i}(u)+C\left[t+\int_{0}^{x} \tilde{v}_{i}^{-1}(u, x) d x\right]\right\} & \text { for } \beta=0,
\end{array}
$$


where $C$ is an arbitrary constant, the integrations with respect to $x$ are performed at a constant value of $u$, and $\left\{w_{i}(u)\right\}$ form an arbitrary solution of the linear system (5.12) with $\Gamma_{i j}^{i}$ defined by (6.8). The extent of arbitrariness is the same as in Theorem 6.1.

6.3. Invariant solutions and linearization. The equations determining invariant solutions of systems (6.1) and (6.7) subject to the constraint $u_{x}^{i} \neq 0$ for $i=1,2, \ldots, n$ are obtained from the Lie equations (6.5) and (6.10) with the invariance condition $u_{\tau}^{i}=0$ : $A_{i}(u, t, x)=0$ and $\tilde{A}_{i}(u, t, x)=0$ with $A_{i}$ and $\tilde{A}_{i}$ defined by (6.6) and (6.11), respectively, where $c=1$ without loss of generality:

$$
\begin{aligned}
w_{i}(u)+\exp \left\{-\beta\left[x+\int_{0}^{t} v_{i}(u, t) d t\right]\right\}=0 & (\beta \neq 0), \\
w_{i}(u)+x+\int_{0}^{t} v_{i}(u, t) d t=0 & (\beta=0), \\
w_{i}(u)+\exp \left\{-\beta\left[t+\int_{0}^{x} \tilde{v}_{i}^{-1}(u, x) d x\right]\right\}=0 & (\beta \neq 0), \\
w_{i}(u)+t+\int_{0}^{x} \tilde{v}_{i}^{-1}(u, x) d x=0 & (\beta=0),
\end{aligned}
$$

with $i=1,2, \ldots, n$ for systems (6.1) and (6.7), respectively. Here the set of functions $w_{i}(u)$ forms an arbitrary smooth solution of the linear system (5.12) with $\Gamma_{i j}^{i}$ defined by (6.3) and (6.8), respectively. Thus, the above equations determine a linearizing transformation for systems (6.1) and (6.7) reducing them to the linear system (5.12). These equations determine the solutions $u^{i}=u^{i}(x, t)$ of the original nonlinear system if the conditions of the implicit function theorem are satisfied. More complete results for diagonal systems with an explicit $t$ - or $x$-dependence and an example of a new integrable system of this class can be found in [13].

7. Conclusions. The existence of an infinite-dimensional group of the hydrodynamic symmetries for the equations of the hydrodynamic type is an important property which provides the existence of linearizing transformations. The reason for this is that the degree of generality of the set of symmetries coincides with the degree of generality of the general solution set for these equations. Therefore, almost all solutions are the invariant solutions and they are obtained by standard formulas provided that the symmetries are already determined. Such a formula gives a linearizing transformation reducing the original nonlinear problem to the linear problem of determining the symmetries. The additional property is the existence of the recursion operator which makes it possible to solve partially the linear problem by constructing infinite discrete sets of its solutions and hence solutions of the original nonlinear equations. The existence of such an operator also has a group-theoretical basis since the recursion operator is completely determined by the symmetries of the determining equations for the hydrodynamic symmetries, that is, by the "symmetries of symmetries."

This shows a group-theoretical origin of linearizing transformations and of the integrability property by which we mean a possibility to construct infinitely many exact solutions. The Hamiltonian structure, if it exists, does not improve the integrability 
properties of equations of the hydrodynamic type. We have to conclude that the symmetry is the major necessary property that insures the integrability which was the original idea of S. Lie.

\section{REFERENCES}

[1] R. L. Anderson and N. H. Ibragimov, Lie-Bäcklund Transformations in Applications, SIAM Studies in Applied Mathematics, vol. 1, Society for Industrial and Applied Mathematics (SIAM), Pennsylvania, 1979.

[2] M. Arik, F. Neyzi, Y. Nutku, P. J. Olver, and J. M. Verosky, Multi-Hamiltonian structure of the Born-Infeld equation, J. Math. Phys. 30 (1989), no. 6, 1338-1344.

[3] V. G. Bagrov, B. F. Samsonov, A. V. Shapovalov, and I. V. Shirokov, Commutative subalgebras of symmetry operators of the wave equation including one second order operator and separation of variables, preprint No. 27, 1990, Tomsk Scientific Center of the Siberian Branch of Academy of Sciences of USSR, Tomsk.

[4] B. A. Dubrovin and S. P. Novikov, Hamiltonian formalism of one-dimensional systems of hydrodynamic type and the Bogolyubov-Whitham averaging method, Soviet Math. Dokl. 27 (1983), 665-669.

[5] _ Hydrodynamics of weakly deformed soliton lattices. Differential geometry and Hamiltonian theory, Russian Math. Surveys 44 (1989), no. 6, 35-124.

[6] D. F. Egorov, Works in Differential Geometry, Izdat. Nauka, Moscow, 1970 (Russian), edited by S. P. Finikov.

[7] E. V. Ferapontov, Compatible Poisson brackets of hydrodynamic type, J. Phys. A 34 (2001), no. 11, 2377-2388.

[8] E. V. Ferapontov, D. A. Korotkin, and V. A. Shramchenko, Boyer-Finley equation and systems of hydrodynamic type, Class. Quantum Grav. 19 (2002), no. 24, L205-L210.

[9] E. V. Ferapontov and M. V. Pavlov, Hydrodynamic reductions of the heavenly equation, Class. Quantum Grav. 20 (2003), no. 11, 2429-2441.

[10] E. V. Ferapontov and S. P. Tsarëv, Systems of hydrodynamic type that arise in gas chromatography. Riemann invariants and exact solutions, Mat. Model. 3 (1991), no. 2, 82-91 (Russian).

[11] B. Fuchssteiner and A. S. Fokas, Symplectic structures, their Bäcklund transformations and hereditary symmetries, Phys. D 4 (1981), no. 1, 47-66.

[12] J. Gibbons and S. P. Tsarëv, Reductions of the Benney equations, Phys. Lett. A 211 (1996), no. 1, 19-24.

[13] A. M. Grundland, M. B. Sheftel, and P. Winternitz, Invariant solutions of hydrodynamic-type equations, J. Phys. A 33 (2000), no. 46, 8193-8215.

[14] H. Gümral and Y. Nutku, Multi-Hamiltonian structure of equations of hydrodynamic type, J. Math. Phys. 31 (1990), no. 11, 2606-2611.

[15] N. H. Ibragimov, Transformation Groups Applied to Mathematical Physics, Mathematics and Its Applications (Soviet Series), D. Reidel Publishing, Dordrecht, 1985.

[16] N. H. Ibragimov and A. B. Shabat, Evolutionary equations with nontrivial Lie-Bäcklund group, Funct. Anal. Appl. 14 (1980), no. 1, 19-28.

[17] _ Infinite Lie-Bäcklund algebras, Funct. Anal. Appl. 14 (1980), no. 4, 313-315.

[18] P. D. Lax, Integrals of nonlinear equations of evolution and solitary waves, Comm. Pure Appl. Math. 21 (1968), 467-490.

[19] F. Magri, A simple model of the integrable Hamiltonian equation, J. Math. Phys. 19 (1978), no. 5, 1156-1162.

[20] Y. I. Manin, Algebraic aspects of nonlinear differential equations, J. Soviet Math. 11 (1979), $1-122$.

[21] A. V. Mikhaĭlov, A. B. Shabat, and V. V. Sokolov, The symmetry approach to classification of integrable equations, What Is Integrability? Springer Ser. Nonlinear Dynam., Springer-Verlag, New York, 1991, pp. 115-184. 
[22] W. Miller, Jr., Symmetry and Separation of Variables, Encyclopedia of Mathematics and Its Applications, vol. 4, Addison-Wesley Publishing, Massachusetts, 1977.

[23] P. J. Olver, Applications of Lie Groups to Differential Equations, Graduate Texts in Mathematics, vol. 107, Springer-Verlag, New York, 1986.

[24] P. J. Olver and Y. Nutku, Hamiltonian structures for systems of hyperbolic conservation laws, J. Math. Phys. 29 (1988), no. 7, 1610-1619.

[25] L. V. Ovsiannikov, Lectures on Foundations of Gas Dynamics, Nauka, Moscow, 1981 (Russian).

[26] M. V. Pavlov, Hamiltonian formalism of the electrophoresis equation. Integrable equations of hydrodynamics, preprint No.17, 1987, Landau Inst. Theor. Phys., Moscow (Russian).

[27] B. L. Roždestvenskiı̆ and N. N. Janenko, Systems of Quasilinear Equations and Their Applications to Gas Dynamics, Translations of Mathematical Monographs, vol. 55, American Mathematical Society, Rhode Island, 1983.

[28] M. B. Sheftel, Linearization of homogeneous quasilinear systems by the method of groupanalysis, differential geometry and Hamiltonian formalism, Lie-Bäcklund Groups and Quasilinear Systems (Leningrad, 1989), LIIAN, preprint No. 106, 4-24, Chapter 1 (Russian).

[29] _ Lie-Bäcklund groups admitted by equations of one-dimensional gas dynamics, Vestnik Leningrad. Univ. Mat. Mekh. Astronom. (1982), no. 7, 37-41 (Russian).

[30] _ On the infinite-dimensional noncommutative Lie-Bäcklund algebra associated with the equations of one-dimensional gas dynamics, Theoret. and Math. Phys. 56 (1983), 878-891.

[31] _ Integration of Hamiltonian systems of hydrodynamic type with two dependent variables with the aid of the Lie-Bäcklund group, Funct. Anal. Appl. 20 (1986), 227-235.

[32] _ Group analysis and linearization of quasilinear systems, Current Group Analysis (Baku, 1988), Èlm, Baku, 1989, pp. 244-250 (Russian).

[33] _ Higher integrals and symmetries of semi-Hamiltonian systems, Differentsial'nye Uravneniya 29 (1993), no. 10, 1782-1795, 1838 (Russian), translated in Differential Equations 29 (1993), no. 10, 1548-1560.

[34] _ Lie-Bäcklund group analysis of two-component systems of hydrodynamic type, Current Group Analysis and Problems of Mathematical Modeling (Samara, 1993), Izdat. Saratov. Univ. Samar. Filial, Samara, 1993, pp. 194-198.

[35] _ Group analysis of defining equations - a method for finding recursion operators, Differentsial'nye Uravneniya 30 (1994), no. 3, 444-456 (Russian).

[36] _ Symmetries, recursions and integrals for hydrodynamic-type systems, Dr. Phys.Math. Sci. Thesis, Tomsk State University, Tomsk, 1994 (Russian).

[37] _ Generalized hydrodynamic-type systems, CRC Handbook of Lie Group Analysis of Differential Equations. Vol. 3, CRC Press, Florida, 1995, pp. 169-189.

[38] V. M. Teshukov, Hyperbolic systems, which admit nontrivial Lie-Bäcklund groups, LieBäcklund Groups and Quasilinear Systems (Leningrad, 1989), LIIAN, preprint No. 106, 25-30 (Russian).

[39] S. P. Tsarëv, On Poisson brackets and one-dimensional systems of hydrodynamic type, Soviet Math. Dokl. 31 (1985), 488-491.

[40] - The geometry of Hamiltonian systems of hydrodynamic type. The generalized hodograph method, Math. USSR-Izv. 37 (1991), no. 2, 397-419.

M. B. Sheftel: Department of Higher Mathematics, North-Western State Technical University, Millionnaya Street 5, St. Petersburg 191186, Russia

Current address: Department of Physics, Boğaziçi University, 34342 Bebek, Istanbul, Turkey Current address: Feza Gursey Institute, P.O. Box 6, 81220 Cengelkoy, Istanbul, Turkey

E-mail address: shefte1@gursey.gov.tr; mikhai1.shefte1@boun.edu.tr 


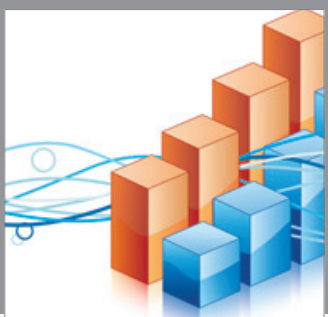

Advances in

Operations Research

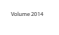

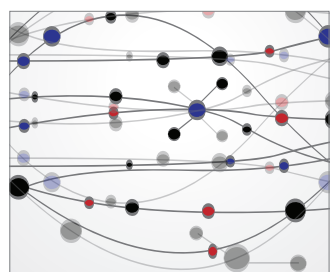

\section{The Scientific} World Journal
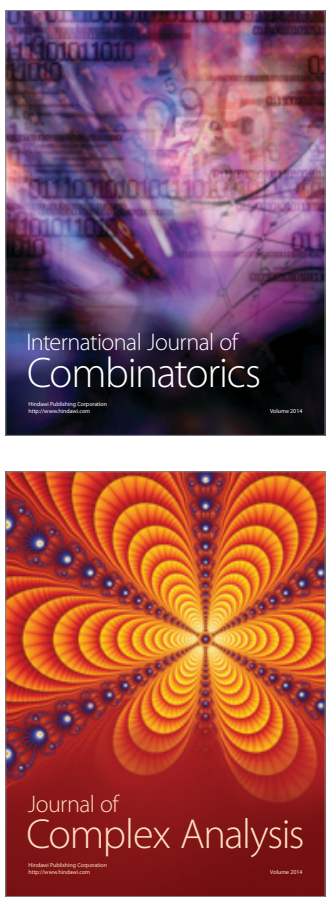

International Journal of

Mathematics and

Mathematical

Sciences
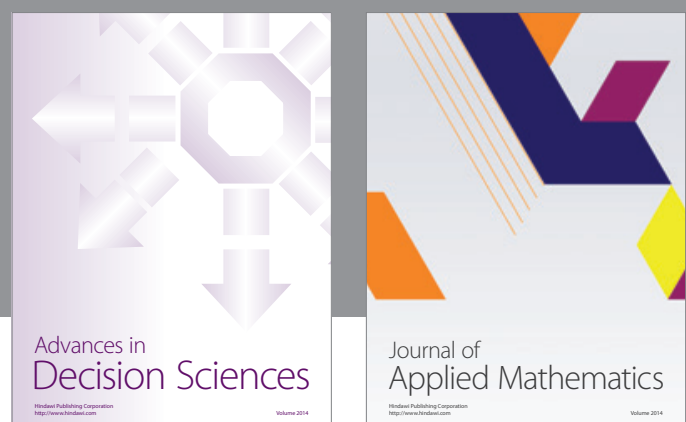

Journal of

Applied Mathematics
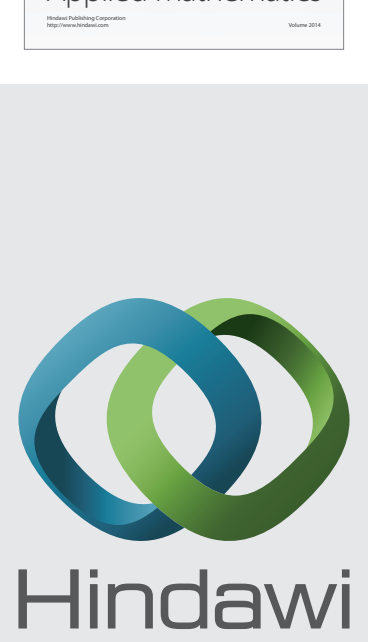

Submit your manuscripts at http://www.hindawi.com
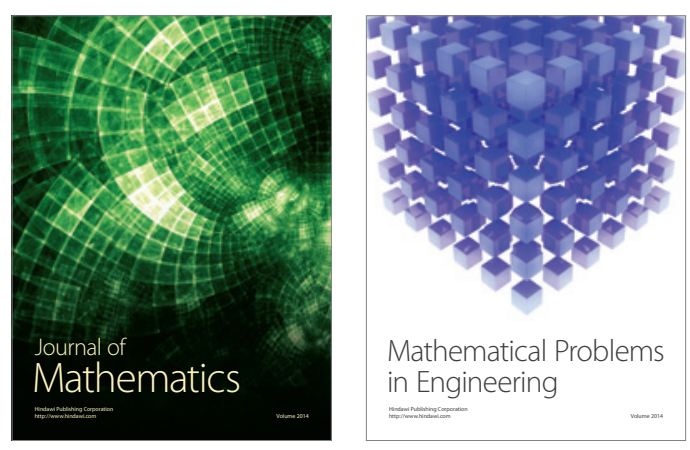

Mathematical Problems in Engineering
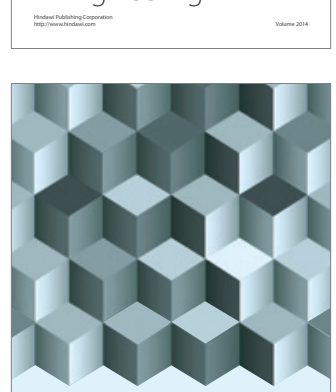

Journal of

Function Spaces
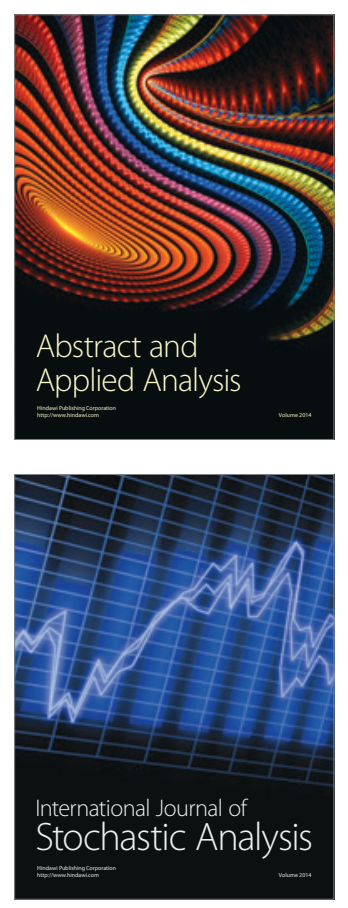

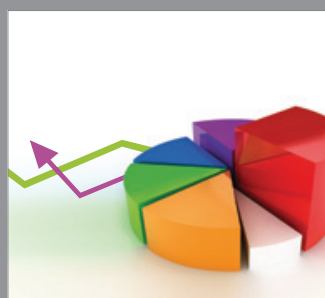

ournal of

Probability and Statistics

Promensencen
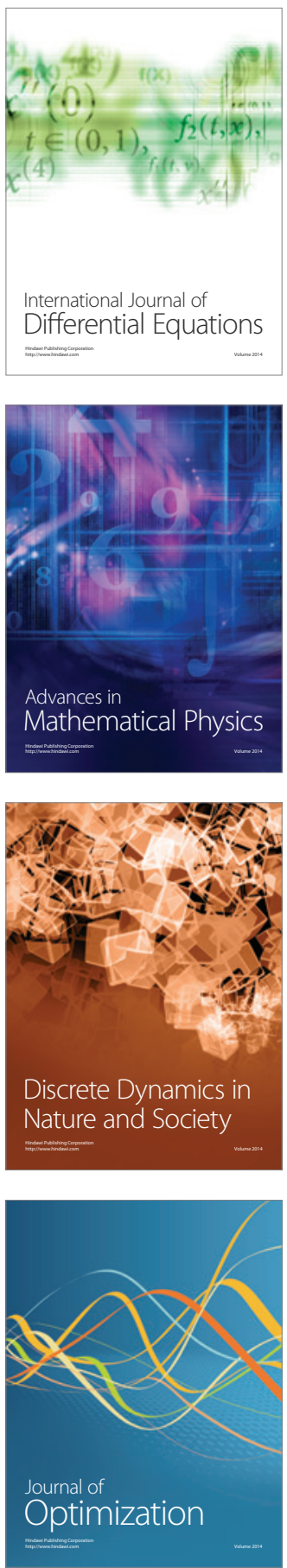